\title{
CONVERGENCE TO EQUILIBRIUM FOR A PHASE-FIELD MODEL FOR THE MIXTURE OF TWO VISCOUS INCOMPRESSIBLE FLUIDS*
}

\author{
LIYUN ZHAO ${ }^{\dagger}$, HAO WU ${ }^{\ddagger}$, AND HAIYANG HUANG $\$$
}

\begin{abstract}
In this paper, we study the existence and long-time behavior of global strong solutions to a system describing the mixture of two viscous incompressible Newtonian fluids of the same density. The system consists of a coupling of Navier-Stokes and Cahn-Hilliard equations. We first show the global existence of strong solutions in several cases. Then we prove that the global strong solution of our system will converge to a steady state as time goes to infinity. We also provide an estimate on the convergence rate.
\end{abstract}

Key words. Navier-Stokes equation, Cahn-Hilliard equation, convergence to equilibrium, Łojasiewicz-Simon approach.

AMS subject classifications. 35Q35, 35K55, 76D05

\section{Introduction}

In this paper, we investigate the following coupled Navier-Stokes/Cahn-Hilliard equations:

$$
\begin{aligned}
\mathbf{u}_{t}+(\mathbf{u} \cdot \nabla) \mathbf{u}+\nabla p-2 \operatorname{div}(\nu(\phi) D(\mathbf{u})) & =-\lambda \nabla \cdot(\nabla \phi \otimes \nabla \phi), \\
\nabla \cdot \mathbf{u} & =0, \\
\phi_{t}+(\mathbf{u} \cdot \nabla) \phi & =\operatorname{div}(\gamma(\phi) \nabla \mu), \\
\text { with } \mu=-\Delta \phi+F^{\prime}(\phi), &
\end{aligned}
$$

for $(x, t) \in \Omega \times(0,+\infty)$. Here, $\Omega \subset \mathbb{R}^{n}(n=2,3)$ is a bounded domain with smooth boundary $\Gamma$. Equation (1.1) is the linear momentum equation, where $\mathbf{u}$ is the velocity field of the mixture, and $\phi$ and $p$ denote the phase function and pressure, respectively. $D(\mathbf{u})=\frac{1}{2}\left(\nabla \mathbf{u}+\nabla \mathbf{u}^{T}\right)$ is the stretching tensor. $\nabla \phi \otimes \nabla \phi$ denotes the induced elastic stress, which is a $n \times n$ matrix whose $(i, j)$-th entry is $\frac{\partial \phi}{\partial x_{i}} \frac{\partial \phi}{\partial x_{j}}$ for $1 \leq i, j \leq n$. Equation (1.2) implies the incompressibility of both fluids in the mixture and equation (1.3) is the phase equation of Cahn-Hilliard type. $\lambda$ is a positive constant which denotes the surface tension. $\nu(\phi)$ and $\gamma(\phi)$ are scalar functions of $\phi$ which represent the kinematic viscosity and the mobility, respectively. We may refer to $[1,3,20,21]$ and the references therein for detailed discussions of the above notations. System (1.1)-(1.3) is subject to the initial data

$$
\left.\mathbf{u}\right|_{t=0}=\mathbf{u}_{0}(x),\left.\quad \phi\right|_{t=0}=\phi_{0}(x), \quad x \in \Omega,
$$

and boundary conditions

$$
\mathbf{u}(x, t)=0, \quad(x, t) \in \Gamma \times(0,+\infty),
$$

*Received: June 4, 2009; accepted (in revised version): September 18, 2009. Communicated by Chun Liu.

${ }^{\dagger}$ School of Mathematical Sciences, Beijing Normal University, Key Laboratory of Mathematics and Complex Systems, MCE, 100875 Beijing, China (zhaoliyun@mail.bnu.edu.cn).

${ }^{\ddagger}$ School of Mathematical Sciences, Fudan University, 200433 Shanghai, China (haowufd@yahoo. com).

${ }^{\S}$ School of Mathematical Sciences, Beijing Normal University, Key Laboratory of Mathematics and Complex Systems, MCE, 100875 Beijing, China (hhywsg@bnu.edu.cn). Corresponding author. 


$$
\partial_{\mathbf{n}} \phi(x, t)=\partial_{\mathbf{n}} \mu(x, t)=0, \quad(x, t) \in \Gamma \times(0,+\infty) .
$$

Here, $\mathbf{n}$ is the outward unit normal to the boundary $\Gamma$. Equation (1.5) is the usual no-slip boundary condition for viscous fluids. $\left.\partial_{\mathbf{n}} \phi\right|_{\Gamma}=0$ describes a contact angle of $\pi / 2$ of the diffused interface and the boundary $\Gamma .\left.\partial_{\mathbf{n}} \mu\right|_{\Gamma}=0$ means that there is no flux of the components through the boundary, and thus the integral of $\phi$ is preserved for $t \geq 0$ (cf. [1, 24]).

Based on an energetic variational approach (EVA), the above system in the case with constant viscosity and mobility was derived in [20] to model the mixture of two fluids. Such an approach can also apply to more general cases with variable density, viscosity and mobility (cf. [1, 21]). In [20], the authors considered a specific type of mixture of two incompressible fluids with the same density and viscosity, which are assumed to be macroscopically immiscible. Instead of the classical sharp interface between both fluids, an interfacial region is imposed, whose length scale is measured by a parameter $\eta>0$ (the width of the interface). Within this "thin" transition region, the fluids are mixed and have to store certain amount of "mixing energy"

$$
W(\phi, \nabla \phi)=\int_{\Omega}\left[\frac{1}{2}|\nabla \phi|^{2}+\frac{1}{\eta^{2}} F(\phi)\right] d x
$$

$\eta$ can be viewed as a parameter indicating the competition between bulk energy $\int_{\Omega} F(\phi) d x$ and the interface surface energy $\int_{\Omega}|\nabla \phi|^{2} d x$. The physical relevant energy density function $F$ usually has a double-well structure, which represents the two phases of the mixture. The most often used one has the following form (cf. [24, 20, $32,34,18,3])$

$$
F(\phi)=\frac{1}{4}\left(\phi^{2}-1\right)^{2}
$$

Besides, in the literature, singular free energy density function like

$$
F(\phi)=\kappa_{0}-\kappa_{1} \phi^{2}+\kappa_{2}((1+\phi) \ln (1+\phi)+(1-\phi) \ln (1-\phi)), \quad \kappa_{i}>0(i=0,1,2)
$$

has also been widely studied (cf. $[1,2,7,3,22]$ ). Comparing with the smooth energy density (1.7), it has the advantage that the phase function will stay in the physical reasonable interval $[-1,1]$.

As far as the mathematical analysis for system (1.1)-(1.3) is concerned, Boyer [3] studied it as a model for the evolution of a binary alloy in a periodical channel under shear. For a suitably smooth double-well potential, he showed the existence of global weak solutions, which are strong and unique with regular initial data if $n=2$ or $n=3$ and $t \in\left(0, T_{0}\right)$ for a sufficiently small $T_{0}>0$. Moreover, the case of the logarithmic potential (1.8) was also considered in connection with a degenerate mobility there. A more complete mathematical theory of existence, uniqueness, regularity and asymptotic behavior of solutions to (1.1)-(1.3) with singular potential (1.8) as well as variable viscosity and constant mobility was given in [1]. In the recent paper [9], the authors considered system (1.1)-(1.6) in 2-D with constant viscosity and mobility. They proved existence of a global attractor as well as an exponential attractor and then showed the upper bound of fractal dimension of the global attractor (cf. [8] for corresponding results on a 2-D Navier-Stokes-Allen-Cahn system). We also notice that in [10], the long-time dynamics of these systems in 3-D was investigated in the framework of trajectory attractors. 
In this paper, we first obtain some useful inequalities, which together with the large viscosity assumption (also large mobility assumption for variable $\gamma$ ) indicate the existence of global strong solutions to problem (1.1)-(1.6) with variable viscosity and mobility in the three dimensional case. This is an extension of the recent result [38] for constant viscosity and mobility. We also discuss the existence of global strong solutions for initial data that are close to the absolute minimizer of the mixing energy in the 3-D case.

Next, we are interested in the asymptotic behavior of strong solutions to system (1.1)-(1.6), namely, whether the global strong solution will converge to an equilibrium as time goes to infinity? How about the convergence rate?

In [3], the author proved an asymptotic stability of a specific constant stationary solution $\left(\mathbf{u}_{\infty}, \phi_{\infty}\right)=\left(0, m\left(\phi_{0}\right)\right)$, where $m\left(\phi_{0}\right)=\frac{1}{|\Omega|} \int_{\Omega} \phi_{0}(x) d x$. It was required that $F$ is convex in a neighborhood of $m\left(\phi_{0}\right)$, in other words, $m\left(\phi_{0}\right)$ should lie in a metastable region of $F$. However, in general it is difficult to obtain the stability result of stationary solutions even for the single Cahn-Hilliard equation in the high dimensional case. On the other hand, in recent years, the so-called Łojasiewicz-Simon approach has been proven to be very useful in the study of long-time behavior of global solutions to nonlinear evolution equations in high dimensional case $(n \geq 2)$. Under the basic assumption that the nonlinear term is analytic in the unknown function, the convergence of uniformly bounded global solutions to equilibria as time goes to infinity can be proven (cf. $[2,5,8,9,17,18,24,25,32,33,34]$ and references therein). In particular, we refer to $[24,32,34]$ for the convergence result concerning the single Cahn-Hilliard equation subject to various boundary conditions. For the system (1.1)(1.3), a first result in this aspect was provided in [1], in the case with a singular free energy density. Due to the regularity of weak solutions for large time (eventually regularity), the author applied the Eojasiewicz-Simon approach to show the convergence to stationary solutions as $t \rightarrow \infty$. However, no estimate on the convergence rate was given there. It is known that an estimate in a certain (lower order) norm can usually be obtained directly from the Eojasiewicz-Simon approach (see, e.g., [14, 40]). Then, one straightforward way to get estimates in higher order norms is to use interpolation inequalities (cf. [14]). We notice that the decay exponent deteriorates in this case.

In this paper, we first apply the Łojasiewicz-Simon approach to prove the convergence to equilibria of global strong solutions to (1.1)-(1.6) in both 2-D and 3-D. Then we show that by using suitable energy estimates and constructing proper differential inequalities, it is possible to obtain the same estimates on convergence rate in both higher and lower order norms. Our approach in some sense improves the previous results in the literature (see, for instance, $[14,40]$ ) and it can apply to many other problems (cf. $[8,9,33,34,35,36,37])$. We mention that in [36], the author studied a simplified system of the Ericksen-Leslie equations for the flow of nematic liquid crystals and achieved the convergence to steady state solutions with convergence rate (cf. also [37] for a more general system). Instead of the Cahn-Hilliard equation (1.3), the system considered there consists of a coupling of Navier-Stokes equation for the velocity field and a second order equation of Ginzburg-Landau type for molecule director (cf. [19]). Using the idea in [19, 36], we introduce a new higher order energy inequality for our system (1.1)-(1.6), which not only yields uniform bounds for the global strong solution but also helps to obtain the convergence rate in a higher order norm. After we finished the present work, we noticed that the convergence of the weak solution to equilibrium was obtained for system (1.1)-(1.6) in 2-D with constant viscosity and mobility in the recent manuscript [9]. However, we are now dealing with 
strong solutions to a more general system (1.1)-(1.6) with variable viscosity/mobility in both 2-D and 3-D, the proof is much more involved.

For viscosity $\nu$ and mobility $\gamma$, we make the following assumptions:

(A1) $\nu \in C^{2}(\mathbb{R}), \nu^{\prime}, \nu^{\prime \prime}$ are bounded on $\mathbb{R}$, and there exists a constant $\nu_{1}>0$ such that $\nu \geq \nu_{1}$

(A2) $\gamma \in C^{2}(\mathbb{R}), \gamma^{\prime}, \gamma^{\prime \prime}$ are bounded on $\mathbb{R}$, and there exists a constant $\gamma_{1}>0$ such that $\gamma \geq \gamma_{1}$.

REMARK 1.1. For the sake of simplicity, we shall just treat the typical physical relevant double-well energy density $F(\phi)=\frac{1}{4}\left(\phi^{2}-1\right)^{2}$. However, under the assumptions (A1) and (A2), it is straightforward to check that our result holds true for more general function $F$ satisfying the following conditions:

(F1) $F(s)$ is analytic in $s \in \mathbb{R}$;

(F2)

$$
\left|F^{\prime \prime \prime}(s)\right| \leq C\left(1+|s|^{q}\right), \quad \forall s \in \mathbb{R},
$$

where $C \geq 0, q \in[0,+\infty)$ for $n=2$ and $q \in[0,2)$ for $n=3$;

(F3)

$$
\liminf _{|s| \rightarrow+\infty} F^{\prime \prime}(s)>0
$$

We relax the assumptions on the viscosity $\nu$ and mobility $\gamma$ such that $\nu$ and $\gamma$ are allowed to have a linear growth. As a result, comparing with the assumptions of $F$ in [3], the growth of $F^{\prime \prime \prime}$ should be weakened (in the 3-D case) to ensure the higher order energy inequality (cf. (2.5)). A careful use of embedding theorems shows that growth assumption (F2) will be enough for the arguments in [3] and thus the original growth assumptions on $F^{\prime}, F^{\prime \prime}$ in [3] (also in [38]) can be relaxed. Assumption (F3) means that the function $F^{\prime}(s)$ is monotonic increasing outside a bounded subset of $\mathbb{R}$. More precisely,

$$
\liminf _{s \rightarrow+\infty} F^{\prime}(s) \geq 1, \quad \liminf _{s \rightarrow-\infty} F^{\prime}(s) \leq-1
$$

Thus $F(s) \geq 0$ outside a bounded subset of $\mathbb{R}$. As a result, the energy functional $E(\mathbf{u}, \phi)$ (cf. (2.1)) is bounded from below (see, for instance, [11, Lemma 3.2] for a detailed proof).

Before stating our main results, we introduce some functional spaces (cf. [1, 3]). As usual, we denote $H_{0}^{1}(\Omega)$ to be the closure of $C_{0}^{\infty}(\Omega)$ in $H^{1}(\Omega)$. Let $C_{0, \text { div }}^{\infty}(\Omega)$ be the space of all divergence free vectors in $\left(C_{0}^{\infty}(\Omega)\right)^{n}(n=2,3)$. We denote by $H$ the closure of $C_{0, \text { div }}^{\infty}(\Omega)$ in $\left(L^{2}(\Omega)\right)^{n}$. Moreover, we set

$$
\begin{aligned}
& V=\left(H_{0}^{1}(\Omega)\right)^{n} \cap H, \\
& V_{2}=\left(H^{2}(\Omega)\right)^{n} \cap\left(H_{0}^{1}(\Omega)\right)^{n} \cap H, \\
& \Phi_{2}=\left\{\phi \in H^{2}(\Omega)\left|\partial_{\mathbf{n}} \phi\right|_{\Gamma}=0\right\}, \\
& \Phi_{4}=\left\{\phi \in H^{4}(\Omega)\left|\partial_{\mathbf{n}} \phi\right|_{\Gamma}=\left.\partial_{\mathbf{n}} \mu\right|_{\Gamma}=0,\right\}, \\
& \mathcal{D}=\left\{\phi \in H^{2}(\Omega)\left|\int_{\Omega} \phi d x=\int_{\Omega} \phi_{0} d x, \partial_{\mathbf{n}} \phi\right|_{\Gamma}=0\right\} .
\end{aligned}
$$

TheOrem 1.1. Suppose $n=2$. Assume that (A1), (A2), and (F1)-(F3) are satisfied. For any initial data $\mathbf{u}_{0} \in V$ and $\phi_{0} \in \Phi_{2}$, system (1.1)-(1.6) admits a unique global 
strong solution $(\mathbf{u}, \phi)$ such that $(\mathbf{u}, \phi)$ converges to a certain equilibrium $\left(0, \phi_{\infty}\right)$ as time goes to infinity:

$$
\lim _{t \rightarrow+\infty}\left(\|\mathbf{u}(\cdot, t)\|_{V}+\left\|\phi(\cdot, t)-\phi_{\infty}\right\|_{H^{3}}\right)=0 .
$$

Here, $\phi_{\infty}$ is a solution to the stationary problem:

$$
\left\{\begin{array}{l}
-\Delta \phi_{\infty}+f\left(\phi_{\infty}\right)=\mu_{\infty}, \quad x \in \Omega, \\
\partial_{n} \phi_{\infty}=0, \quad x \in \Gamma, \\
\int_{\Omega} \phi_{\infty} d x=\int_{\Omega} \phi_{0} d x,
\end{array}\right.
$$

where

$$
f=F^{\prime}
$$

and $\mu_{\infty}$ is a constant such that

$$
\mu_{\infty}=\frac{1}{|\Omega|} \int_{\Omega} f\left(\phi_{\infty}\right) d x .
$$

Moreover, we have the following estimate on the convergence rate:

$$
\|\mathbf{u}(\cdot, t)\|_{V}+\left\|\phi(\cdot, t)-\phi_{\infty}\right\|_{H^{3}} \leq C(1+t)^{-\theta /(1-2 \theta)}, \quad \forall t \geq 1 .
$$

$C \geq 0$ is a constant depending on $\left\|\mathbf{u}_{0}\right\|_{V},\left\|\phi_{0}\right\|_{\Phi_{2}}, \Omega, \phi_{\infty}, \nu_{1}, \gamma_{1}$ and $\lambda . \theta \in\left(0, \frac{1}{2}\right)$ is a constant depending on $\phi_{\infty}$.

REMARK 1.2. Notice that (1.10) is the stationary Cahn-Hilliard equation with Neumann boundary condition. Non-constant solutions of (1.10) have been studied extensively, and various types of solution such as spike-like solutions and bubble solutions have been constructed (see, for example, $[4,6,29,30,31]$ and the references therein). For the Cahn-Hilliard equation, further results can be obtained in the 1-D case, for instance, the number of the steady states (i.e., solutions of (1.10)) can be counted and then the convergence to equilibrium can be proven, because the time dependent Cahn-Hilliard equation defines a gradient system in $H^{-1}(\Omega)$ (cf. $[12,23,39]$ ).

When the spatial dimension is three, due to the difficulties from Navier-Stokes equation as well as Cahn-Hilliard equation with nonconstant mobility, we cannot expect the same result as in 2-D. In what follows, we deal with some cases, in which the global strong solutions will exist. The first result is concerning the large viscosity (also large mobility) case:

TheOREM 1.3. Suppose $n=3$.

(1) Assume that $\gamma>0$ is a constant and (A1), (F1)-(F3) are satisfied. For any $\mathbf{u}_{0} \in V, \phi_{0} \in \Phi_{2}$, system (1.1)-(1.6) admits a unique global strong solution $(\mathbf{u}, \phi)$ under the large viscosity assumption $\nu \geq \nu_{1} \geq \nu_{0}\left(\lambda, \gamma, \mathbf{u}_{0}, \phi_{0}\right)$.

(2) Assume that (A1), (A2) and (F1)-(F3) are satisfied. For any $\mathbf{u}_{0} \in V, \phi_{0} \in$ $\Phi_{2}$, system (1.1)-(1.6) admits a unique global strong solution $(\mathbf{u}, \phi)$ provided that the viscosity and mobility are properly large such that $\nu \geq \nu_{1} \geq \nu_{0}\left(\lambda, \mathbf{u}_{0}, \phi_{0}\right)$ and $\gamma \geq \gamma_{1} \geq$ $\gamma_{0}\left(\lambda, \mathbf{u}_{0}, \phi_{0}\right)$.

In the above two cases, the unique global strong solution enjoys the same asymptotic behavior as in Theorem 1.1. 
The second one is a "stability" result for the near equilibrium initial data:

THEOREM 1.4. Suppose that $n=3$ and (A1), (A2), (F1)-(F3) are satisfied. Let $\phi^{*} \in \mathcal{D}$ be an absolute minimizer of the functional

$$
\mathcal{E}(\phi)=\frac{1}{2}\|\nabla \phi\|^{2}+\int_{\Omega} F(\phi) d x
$$

in the sense that $\mathcal{E}\left(\phi^{*}\right) \leq \mathcal{E}(\phi)$ for all $\phi \in \mathcal{D}$. For any $\mathbf{u}_{0} \in V, \phi_{0} \in \Phi_{2} \cap H^{3}(\Omega)$, there is a constant $\sigma$ which may depend on $\lambda, \gamma_{1}, \nu_{1}, f, \phi^{*}$ and $\left\|\mathbf{u}_{0}\right\|_{V},\left\|\phi_{0}\right\|_{H^{3}}$, such that if $\left\|\mathbf{u}_{0}\right\|+\left\|\phi_{0}-\phi^{*}\right\|_{H^{1}}<\sigma$, then the problem (1.1)-(1.6) admits a unique global strong solution, which enjoys the same asymptotic behavior as in Theorem 1.1.

REMARK 1.5. It is well known that the topology of equilibria set can be rather complicated in the high dimensional case such that it may form a continuum (cf. $[13,24])$. Thus, even if the initial data is sufficiently close to an absolute minimizer of the functional $\mathcal{E}$, the global solution will converge to an equilibrium that may not necessarily be the original minimizer. The result, Theorem 1.4, is in some sense different from the usual concept of stability.

REMARK 1.6. Our results hold true for all parameters $\eta>0$, so we just set $\eta=1$ in the following text. It would be interesting to ask whether these results can be passed to the limit case as $\eta \rightarrow 0$. This goal is out of reach under our current approach.

REMARK 1.7. For the singular free energy density function $F$ like (1.8), whose singular points are $\{-1,1\}$, it has been shown that (cf. [1, Lemma 7.2]) the "separation" property holds for the phase function. Namely, there exist some $T>0$ and $\delta \in(0,1)$ such that when $t>T, \phi(x, t) \in(-1+\delta, 1-\delta)$. As a result, the singular function $F(\phi)$ can be replaced by a bounded smooth function $\tilde{F}(\phi)$ such that $\left.F(\phi)\right|_{[-1+\delta, 1-\delta]}=$ $\left.\tilde{F}(\phi)\right|_{[-1+\delta, 1-\delta]}$. Hence, our arguments for convergence rate may also apply to this case.

The remaining part of this paper is organized as follows. In section 2 , we recall some basic results in the literature. In section 3 , we prove the global existence of strong solutions in 3-D, provided that the viscosity and mobility are properly large. In section 4, we derive a new high order energy inequality and study the global existence of strong solutions in 3-D for initial data that are close to equilibrium. The final section 5 is devoted to the convergence to equilibrium of global strong solutions and the estimates on convergence rate.

\section{Preliminaries}

For a Banach space $X$, we denote its norm by $\|\cdot\|_{X}$. For the sake of simplicity, we use $\|\cdot\|$ and $(\cdot, \cdot)$ for the norm and the inner product on $L^{2}(\Omega)\left(\right.$ or $\left.\left(L^{2}(\Omega)\right)^{n}\right)$, respectively.

Given $\phi \in L^{1}(\Omega)$, we denote its mean value by

$$
m(\phi)=\frac{1}{|\Omega|} \int_{\Omega} \phi d x .
$$

Set $L_{0}^{2}(\Omega)=\left\{\phi \in L^{2}(\Omega): m(\phi)=0\right\}$. We then denote $H_{*}^{1}(\Omega)=H^{1}(\Omega) \cap L_{0}^{2}(\Omega)$, and let $H^{-1}(\Omega)$ be the dual space of $H_{*}^{1}(\Omega)$. We equip $H_{*}^{1}(\Omega)$ with the inner product $(u, v)_{H_{*}^{1}}=(\nabla u, \nabla v)$. Denote $-\Delta_{N}$ to be the (negative) Laplace operator with homogeneous Neumann boundary condition. Then we have (cf. $[1,2])$

$$
<\left(-\Delta_{N}\right) u, v>_{H^{-1}, H_{*}^{1}}=\int_{\Omega}\left(-\Delta_{N}\right) u v d x=(u, v)_{H_{*}^{1}}=(\nabla u, \nabla v), \quad u, v \in H_{*}^{1},
$$




$$
(u, v)_{H^{-1}}=\left(\nabla\left(-\Delta_{N}\right)^{-1} u, \nabla\left(-\Delta_{N}\right)^{-1} v\right) .
$$

Next, we introduce the Stokes operator $S$, which is an unbounded operator with domain $D(S)=V_{2}$ defined by $S \mathbf{u}=-\Delta \mathbf{u}+\nabla \pi \in H$, for all $\mathbf{u} \in V_{2}$, where $\pi \in H^{1}(\Omega)$. Then the following property holds:

Lemma 2.1. (cf. [27, 3]) There exists a constant $C>0$ such that for any $\mathbf{u} \in V_{2}$, it holds

$$
\|\mathbf{u}\|_{V_{2}} \leq C\|S \mathbf{u}\|, \quad\|\pi\|_{H^{1}(\Omega) \backslash \mathbb{R}} \leq C\|S \mathbf{u}\|, \quad\|\pi\|_{L^{2}(\Omega) \backslash \mathbb{R}} \leq C\|\mathbf{u}\|_{V} .
$$

LEMma 2.2. [Korn's inequality] (cf. [15]) Let $\Omega$ be an open and regular domain in $\mathbb{R}^{n}$ and let $\mathbf{u} \in\left(H_{0}^{1}(\Omega)\right)^{n}$ be a vector field on $\Omega$. We have

$$
\|\nabla \mathbf{u}\|^{2} \leq 2\|D(\mathbf{u})\|^{2}
$$

with the equality if we suppose divu $=0$.

Integrating (1.3) over $\Omega$ and using the boundary condition, it is easy to see that $m(\phi(t))$ is conserved for all time.

The total energy of system (1.1)-(1.6) is defined by

$$
E(\mathbf{u}, \phi)=\int_{\Omega}\left(\frac{1}{2}|\mathbf{u}|^{2}+\frac{\lambda}{2}|\nabla \phi|^{2}+\lambda F(\phi)\right) d x .
$$

Problem (1.1)-(1.6) is a dissipative system, such that the following basic energy law holds (cf. $[3,20,38])$ :

$$
\frac{d}{d t} E(\mathbf{u}, \phi)=-\int_{\Omega}\left(2 \nu(\phi)|D(\mathbf{u})|^{2}+\lambda \gamma(\phi)|\nabla \mu|^{2}\right) d x .
$$

Hereafter in this section, we recall some results on the solutions to (1.1)-(1.6) which easily follow from the argument in [3].

First, from the basic energy law (2.2), we can easily see that for weak solutions to system (1.1)-(1.6), there holds

Lemma 2.3. Suppose (F1)-(F3). Let $\mathbf{u}_{0} \in H$ and $\phi_{0} \in H^{1}(\Omega)$. Then

$$
\|\mathbf{u}(t)\|+\|\phi(t)\|_{H^{1}} \leq C, \quad \forall t \geq 0,
$$

where $C$ is a constant depending on $\left\|\mathbf{u}_{0}\right\|,\left\|\phi_{0}\right\|_{H^{1}}$ and $|\Omega|$.

Denote

$$
\begin{aligned}
& \mathcal{A}(t)=\|\nabla \mathbf{u}(t)\|^{2}+\|\Delta \phi(t)\|^{2}, \\
& \mathcal{B}(t)=\|\nabla \mathbf{u}(t)\|^{2}+\|\nabla \Delta \phi(t)\|^{2}+1, \\
& \mathcal{C}(t)=\|S \mathbf{u}(t)\|^{2}+\left\|\Delta^{2} \phi(t)\right\|^{2} .
\end{aligned}
$$

By using the uniform estimate (2.3) and a slight modification of the argument in [3], one can obtain the following energy inequalities.

Lemma 2.4. Assume that (F1)-(F3) are satisfied. Let $\mathbf{u}_{0} \in V$ and $\phi_{0} \in \Phi_{2}$. When $n=2$, the following lemma holds.

$$
\frac{d}{d t} \mathcal{A}(t)+\alpha \mathcal{C}(t) \leq C \mathcal{A}(t) \mathcal{B}(t)+C .
$$


When $n=3$,

$$
\frac{d}{d t} \mathcal{A}(t)+\alpha \mathcal{C}(t) \leq C \mathcal{A}(t) \mathcal{B}(t)+C \mathcal{A}^{5}(t)+C .
$$

Here, $\alpha>0$ is a small constant depending on $\nu_{1}$ and $\gamma_{1}$, and $C$ is a constant depending on $\left\|\mathbf{u}_{0}\right\|,\left\|\phi_{0}\right\|_{H^{1}}$, and $|\Omega|$

Based on Lemma 2.4, a similar argument to that in [3] yields the following wellposedness result on strong solutions to (1.1)-(1.6):

Proposition 2.5. Assume that $\Omega \subset \mathbb{R}^{n}(n=2,3)$ is a bounded domain with smooth boundary and (A1), (A2), (F1)-(F3) are satisfied. Let $\mathbf{u}_{0} \in V$ and $\phi_{0} \in \Phi_{2}$. Then

(a) in the 2-D case, there exists a unique global strong solution to (1.1)-(1.6) such that

$$
\mathbf{u} \in C([0, \infty) ; V) \cap L^{2}\left(0, \infty ; V_{2}\right), \quad \phi \in C\left([0, \infty) ; \Phi_{2}\right) \cap L^{2}\left(0, \infty ; \Phi_{4}\right) .
$$

Moreover, the following uniform estimate holds:

$$
\|\mathbf{u}(t)\|_{V}^{2}+\|\phi(t)\|_{\Phi_{2}}^{2} \leq C, \quad \forall t \geq 0,
$$

where $C$ is a constant depending on $\left\|\mathbf{u}_{0}\right\|_{V},\left\|\phi_{0}\right\|_{\Phi_{2}}$ and $|\Omega|$.

(b) in the 3-D case, there exists a certain (small) $T_{0}>0$ depending on $\left\|\mathbf{u}_{0}\right\|_{V}$, $\left\|\phi_{0}\right\|_{\Phi_{2}}$, and $|\Omega|$ such that there is a unique strong solution to (1.1)-(1.6) on the finite time interval $\left[0, T_{0}\right)$.

REMARK 2.1. Since the value of $\lambda$ does not play an important role in the proof, for the sake of simplicity, we shall always assume that $\lambda=1$ in the remaining part of this paper.

\section{Global Wellposedness under large viscosity assumption}

Recently, the authors in [38] studied the global existence of strong solutions to (1.1)-(1.6) with constant viscosity and mobility in 3-D. Different from the local existence in 3-D as in [3] (see also Proposition 2.5(b)), the global existence of strong solutions was proven in [38], provided that the viscosity $\nu$ is supposed to be properly large.

We shall extend their result to the case with variable viscosity and (nondegenerate) mobility. Similar to $[3,38]$, the following estimates are made for the Garlerkin approximation of weak solutions. We can see that the entire calculation is identical to that as we work with classical (smooth) solutions to (1.1)-(1.6) (cf. also [19]). Hence, we shall just perform the estimates for smooth solutions.

Denote

$$
\tilde{A}(t)=\|\nabla \mathbf{u}(t)\|^{2}+\|\Delta \phi(t)\|^{2}+1=\mathcal{A}(t)+1 .
$$

Lemma 3.1. Suppose that $n=3$ and (A1), (A2), (F1)-(F3) are satisfied. There holds

$$
\frac{1}{2} \frac{d}{d t} \tilde{A}(t)+\left(\nu_{1}-C_{1} \tilde{A}(t)\right)\|S \mathbf{u}(t)\|^{2}+\left(\gamma_{1}-C_{2}\|\Delta \phi\|^{2}-C_{3}\right)\left\|\Delta^{2} \phi(t)\right\|^{2} \leq C_{4} \tilde{A}(t) .
$$

Here, $C_{i}(i=1, \ldots, 4)$ are constants depending only on $\left\|\mathbf{u}_{0}\right\|,\left\|\phi_{0}\right\|_{H^{1}}$ and $|\Omega|$. 
Proof. We multiply (1.1) by $S \mathbf{u}$ and multiply (1.3) by $\Delta^{2} \phi$. Addition of the two resultants and integration over $\Omega$ yield

$$
\begin{aligned}
& \frac{1}{2} \frac{d}{d t}\left(\|\nabla \mathbf{u}\|^{2}+\|\Delta \phi\|^{2}\right)+\int_{\Omega} \nu(\phi)|S \mathbf{u}|^{2} d x+\int_{\Omega} \gamma(\phi)\left|\Delta^{2} \phi\right|^{2} d x \\
= & -((\mathbf{u} \cdot \nabla) \mathbf{u}, S \mathbf{u})-(\Delta \phi \nabla \phi, S \mathbf{u})+(\nu(\phi) \nabla \pi, S \mathbf{u})+2\left(\nu^{\prime}(\phi) \nabla \phi D(\mathbf{u}), S \mathbf{u}\right) \\
& -\left((\mathbf{u} \cdot \nabla) \phi, \Delta^{2} \phi\right)+\left(\gamma(\phi) \Delta f(\phi), \Delta^{2} \phi\right)-\left(\gamma^{\prime}(\phi) \nabla \phi \nabla \Delta \phi, \Delta^{2} \phi\right) \\
& +\left(\gamma^{\prime}(\phi) \nabla \phi \nabla f(\phi), \Delta^{2} \phi\right) .
\end{aligned}
$$

Now we estimate the right hand side term by term. Notice that the following inequalities hold

$$
\begin{aligned}
\|\phi\|_{L^{\infty}} & \leq C\|\phi\|_{H^{1}}^{\frac{1}{2}}\|\phi\|_{H^{2}}^{\frac{1}{2}} \leq C\left(\|\Delta \phi\|^{\frac{1}{2}}+1\right), \\
\|\phi\|_{L^{\infty}} & \leq C\|\phi\|_{H^{1}}^{\frac{5}{6}}\left\|\Delta^{2} \phi\right\|^{\frac{1}{6}}+C\|\phi\|_{H^{1}} \leq C\left(\left\|\Delta^{2} \phi\right\|^{\frac{1}{6}}+1\right), \\
\|\nabla \phi\|_{L^{4}} & \leq C\|\nabla \phi\|^{\frac{3}{4}}\left\|\Delta^{2} \phi\right\|^{\frac{1}{4}}+C\|\nabla \phi\| \leq C\left(\left\|\Delta^{2} \phi\right\|^{\frac{1}{4}}+1\right), \\
\|\nabla \phi\|_{L^{\infty}} & \leq C\left\|\Delta^{2} \phi\right\|^{\frac{1}{2}}\|\nabla \phi\|^{\frac{1}{2}}+C\|\nabla \phi\| \leq C\left(\left\|\Delta^{2} \phi\right\|^{\frac{1}{2}}+1\right), \\
\|\nabla \phi\|_{L^{\infty}} & \leq C\|\nabla \phi\|_{H^{1}}^{\frac{1}{2}}\|\nabla \phi\|_{H^{2}}^{\frac{1}{2}} \leq C\left(\|\nabla \Delta \phi\|^{\frac{1}{2}}\|\Delta \phi\|^{\frac{1}{2}}+\|\Delta \phi\|+1\right), \\
\|\Delta \phi\| & \leq C\|\nabla \phi\|^{\frac{2}{3}}\left\|\Delta^{2} \phi\right\|^{\frac{1}{3}}+C\|\nabla \phi\| \leq C\left(\left\|\Delta^{2} \phi\right\|^{\frac{1}{3}}+1\right), \\
\|\nabla \Delta \phi\| & \leq C\left(\left\|\Delta^{2} \phi\right\|^{\frac{1}{2}}\|\Delta \phi\|^{\frac{1}{2}}+\left\|\Delta^{2} \phi\right\|\right),
\end{aligned}
$$

where $C>0$ is a constant depending on $\|\phi\|_{H^{1}}$ and $|\Omega|$ at most. By Lemma 2.1, Lemma 2.3 and the assumptions (A1), (A2), we have

$$
\begin{aligned}
|((\mathbf{u} \cdot \nabla) \mathbf{u}, S \mathbf{u})| & \leq\|\mathbf{u}\|_{L^{4}(\Omega)}\|\nabla \mathbf{u}\|_{L^{4}(\Omega)}\|S \mathbf{u}\| \leq C\|\mathbf{u}\|^{\frac{1}{4}}\|\nabla \mathbf{u}\|^{\frac{3}{4}}\|\nabla \mathbf{u}\|^{\frac{1}{4}}\|S \mathbf{u}\|^{\frac{7}{4}} \\
& \leq C\|\nabla \mathbf{u}\| \frac{6}{7}\|S \mathbf{u}\|^{2}+C\|\mathbf{u}\|^{2}\|\nabla \mathbf{u}\|^{2} \\
& \leq C\left(\|\nabla \mathbf{u}\|^{2}+1\right)\|S \mathbf{u}\|^{2}+C\|\nabla \mathbf{u}\|^{2}, \\
|(\Delta \phi \nabla \phi, S \mathbf{u})| & \leq\|\Delta \phi\|\|\nabla \phi\|_{L^{\infty}}\|S \mathbf{u}\| \leq C\left(\left\|\Delta^{2} \phi\right\|^{\frac{1}{3}}+1\right)\left(\left\|\Delta^{2} \phi\right\|^{\frac{1}{2}}+1\right)\|S \mathbf{u}\| \\
& \leq C\left\|\Delta^{2} \phi\right\|^{2}+C\|S \mathbf{u}\|^{2}+C, \\
|(\nu(\phi) \nabla \pi, S \mathbf{u})| & \leq C\left(1+\|\phi\|_{L^{\infty}}\right)\|\nabla \pi\|\|S \mathbf{u}\| \leq C\left(1+\|\Delta \phi\|^{\frac{1}{2}}\right)\|S \mathbf{u}\|^{2}, \\
\left|2\left(\nu^{\prime}(\phi) \nabla \phi D(\mathbf{u}), S \mathbf{u}\right)\right| & \leq C\|\nabla \phi\|_{L^{\infty}}\|\nabla \mathbf{u}\|\|S \mathbf{u}\| \leq C\left(\left\|\Delta^{2} \phi\right\|^{\frac{1}{2}}+1\right)\|\nabla \mathbf{u}\|\|S \mathbf{u}\| \\
& \leq C\left\|\Delta^{2} \phi\right\|^{2}+C\|\nabla \mathbf{u}\|^{2}\|S \mathbf{u}\|^{2}+C, \\
\left|\left((\mathbf{u} \cdot \nabla) \phi, \Delta^{2} \phi\right)\right| & \leq\|\mathbf{u}\|\|\nabla \phi\|_{L^{\infty}(\Omega)}\left\|\Delta^{2} \phi\right\| \leq C\left(\left\|\Delta^{2} \phi\right\|^{\frac{1}{2}}+1\right)\left\|\Delta^{2} \phi\right\| \\
& \leq C\left\|\Delta^{2} \phi\right\|^{2}+C, \\
\left|\gamma(\phi)\left(\Delta f(\phi), \Delta^{2} \phi\right)\right| & \leq C\left(1+\|\phi\|_{L^{\infty}}\right)\|\Delta f(\phi)\|\left\|\Delta^{2} \phi\right\| \\
& \leq C\left(1+\|\phi\|_{L^{\infty}}\right)\left(\left\|f^{\prime}(\phi)\right\|_{L^{\infty}}\|\Delta \phi\|+\left\|f^{\prime \prime}(\phi)\right\|_{L^{\infty}}\|\nabla \phi\|_{L^{4}}^{2}\right)\left\|\Delta^{2} \phi\right\| \\
& \leq C\left(1+\|\phi\|_{L^{\infty}}^{3}\right)\|\Delta \phi\|\left\|\Delta^{2} \phi\right\|+C\left(1+\|\phi\|_{L^{\infty}}^{2}\right)\|\nabla \phi\|_{L^{4}}^{2}\left\|\Delta^{2} \phi\right\| \\
& \leq C\left(1+\left\|\Delta^{2} \phi\right\|^{\frac{1}{2}}\right)\left(1+\left\|\Delta^{2} \phi\right\|^{\frac{1}{3}}\right)\left\|\Delta^{2} \phi\right\| \\
& \leq C\left\|\Delta^{2} \phi\right\|^{2}+C, \\
\left|\left(\gamma^{\prime}(\phi) \nabla \phi \nabla \Delta \phi, \Delta^{2} \phi\right)\right| & \leq C\|\nabla \phi\|_{L^{\infty}}\|\nabla \Delta \phi\|\left\|\Delta^{2} \phi\right\| \\
& \leq C\left(\|\nabla \Delta \phi\|^{\frac{1}{2}}\|\Delta \phi\|^{\frac{1}{2}}+\|\Delta \phi\|+1\right)\|\nabla \Delta \phi\|\left\|\Delta^{2} \phi\right\| \\
& \leq C\left\|\Delta^{2} \phi\right\|^{\frac{7}{4}}\|\Delta \phi\|^{\frac{5}{4}}+C\|\Delta \phi\|^{2}\left\|\Delta^{2} \phi\right\|+C\left\|\Delta^{2} \phi\right\|^{\frac{3}{2}}\|\Delta \phi\|^{\frac{3}{2}}
\end{aligned}
$$




$$
\begin{aligned}
& \leq C\left\|\Delta^{2} \phi\right\|^{2}+C\|\Delta \phi\|^{2}\left\|\Delta^{2} \phi\right\|^{2}+C\|\Delta \phi\|^{2}+C, \\
\left|\left(\gamma^{\prime}(\phi) \nabla \phi \nabla f(\phi), \Delta^{2} \phi\right)\right| & =\left|\left(\gamma^{\prime}(\phi) f^{\prime}(\phi)|\nabla \phi|^{2}, \Delta^{2} \phi\right)\right| \leq C\left\|f^{\prime}(\phi)\right\|_{L^{\infty}}\|\nabla \phi\|_{L^{4}}^{2}\left\|\Delta^{2} \phi\right\| \\
& \leq C\left(\left\|\Delta^{2} \phi\right\|^{\frac{1}{3}}+1\right)\left(\left\|\Delta^{2} \phi\right\|^{\frac{1}{2}}+1\right)\left\|\Delta^{2} \phi\right\| \\
& \leq C\left\|\Delta^{2} \phi\right\|^{2}+C .
\end{aligned}
$$

Collecting the above estimates, we infer from (3.2) that (3.1) holds.

If the mobility $\gamma$ is assumed to be a positive constant, by a modification of the above proof we obtain

Lemma 3.2. Suppose that $n=3$, the mobility $\gamma$ is a positive constant and (A1), (F1)-(F3) are satisfied. There holds

$$
\frac{1}{2} \frac{d}{d t} \tilde{A}(t)+\left(\nu_{1}-\tilde{C}_{1} \tilde{A}(t)\right)\|S \mathbf{u}(t)\|^{2}+\frac{\gamma}{2}\left\|\Delta^{2} \phi(t)\right\|^{2} \leq \tilde{C}_{2} \tilde{A}(t) .
$$

Here, $\tilde{C}_{1}, \tilde{C}_{2}$ are constants depending on $\left\|\mathbf{u}_{0}\right\|,\left\|\phi_{0}\right\|_{H^{1}}$, and $|\Omega|$, and $\tilde{C}_{2}$ also depends on $\gamma$.

Based on Lemma 3.1 and Lemma 3.2 respectively, one can use the argument in $[19,37,38]$ to conclude the existence and uniqueness of global strong solutions to system (1.1)-(1.6). The crucial step is to prove the uniform bound of $\widetilde{A}(t)$ such that

$$
\tilde{A}(t) \leq C, \quad \forall t \geq 0 .
$$

For the reader's convenience, we shall sketch the proof here for the case in Lemma 3.1. Without loss of generality, we assume that $\nu_{1} \geq 1, \gamma_{1} \geq 1$. By Lemma 2.2 and (2.2), one can see that

$$
\int_{t}^{t+1}\|\nabla \mathbf{u}(\tau)\|^{2} d \tau \leq C, \quad \forall t \geq 0
$$

and

$$
\int_{t}^{t+1}\|\nabla \mu(\tau)\|^{2} d \tau \leq C, \quad \forall t \geq 0 .
$$

By (2.3), (F2), Poincaré's inequality, the Sobolev embedding theorem, and the Young inequality, we have

$$
\begin{aligned}
\|\Delta \phi\| & \leq\|\mu\|+\|f(\phi)\| \leq C\|\nabla \mu\|+C|m(\mu)|+\|f(\phi)\| \\
& \leq C\|\nabla \mu\|+C\left\|\phi^{4}\right\|_{L^{1}}+C\left\|\phi^{4}\right\|+C \leq C\|\nabla \mu\|+C\|\phi\|_{L^{8}}^{4}+C \\
& \leq C\|\nabla \mu\|+C\left(\|\Delta \phi\|^{\frac{1}{2}}+1\right)+C \leq C\|\nabla \mu\|+\frac{1}{2}\|\Delta \phi\|+C,
\end{aligned}
$$

which indicates that

$$
\int_{t}^{t+1}\|\Delta \phi(\tau)\|^{2} d \tau \leq C, \quad \forall t \geq 0
$$

As a result,

$$
\int_{t}^{t+1} \tilde{A}(\tau) d \tau \leq \int_{t}^{t+1} \mathcal{A}(\tau) d \tau+1 \leq M, \quad \forall t \geq 0,
$$


where $M \geq 0$ is a constant depending on $\left\|\mathbf{u}_{0}\right\|,\left\|\phi_{0}\right\|_{H^{1}}$, and $|\Omega|$.

Take

$$
\nu_{1} \geq 2 C_{1}\left(\tilde{A}(0)+2 C_{4} M+4 M\right)+1, \quad \gamma_{1} \geq 2 C_{2}\left(\tilde{A}(0)+2 C_{4} M+4 M\right)+2 C_{3}+1 .
$$

Since

$$
\nu_{1}>C_{1} \tilde{A}(0), \quad \gamma_{1}>C_{2} \tilde{A}(0)+C_{3},
$$

there exists certain $T_{0}>0$ such that for $t \in\left[0, T_{0}\right]$,

$$
\nu_{1} \geq C_{1} \tilde{A}(t), \quad \gamma_{1} \geq C_{2} \tilde{A}(t)+C_{3} \geq C_{2}\|\Delta \phi(t)\|^{2}+C_{3},
$$

and thus

$$
\frac{d}{d t} \tilde{A}(t) \leq 2 C_{4} \tilde{A}(t)
$$

Let $T^{*}=\sup T_{0}$. We claim that $T^{*} \geq 1$. If not, we have

$$
\tilde{A}\left(T^{*}\right) \leq \tilde{A}(0)+2 C_{4} \int_{0}^{1} \tilde{A}(\tau) d \tau \leq \tilde{A}(0)+2 C_{4} M .
$$

Then we can see that (cf. (3.21))

$$
\nu_{1}>C_{1} \tilde{A}\left(T^{*}\right), \quad \gamma_{1}>C_{2} \tilde{A}\left(T^{*}\right)+C_{3},
$$

which contradict the definition of $T^{*}$.

If $T^{*}<+\infty$, it follows from (3.20) that there is a $t^{*} \in\left[T^{*}-\frac{1}{2}, T^{*}\right]$ such that

$$
\tilde{A}\left(t^{*}\right) \leq 4 M \text {. }
$$

Then

$$
\tilde{A}\left(T^{*}\right) \leq \tilde{A}\left(t^{*}\right)+2 C_{4} \int_{t^{*}}^{T^{*}} \tilde{A}(\tau) d \tau \leq 4 M+2 C_{4} M,
$$

which again implies (3.24). This leads to a contradiction and we conclude that $T^{*}=$ $+\infty$. Summing up, for all $t \geq 0,(3.22)$ is satisfied, namely, $\tilde{A}(t)$ is uniformly bounded. The proof is complete.

REMARK 3.1. We remark that in the above argument the choice of lower bounds for $\nu_{1}, \gamma_{1}$ (cf. (3.21)) is actually not optimal but it is sufficient for the global existence of strong solutions.

The proof for the case in Lemma 3.2 is simpler, so we omit it here. From the above discussion, we can prove the following theorem:

TheOREM 3.2. Suppose $n=3$.

(a) Assume that $\gamma>0$ is a constant and (A1), (F1)-(F3) are satisfied. For any $\mathbf{u}_{0} \in V, \phi_{0} \in \Phi_{2}$, system (1.1)-(1.6) admits a unique global strong solution $(\mathbf{u}, \phi)$ under the large viscosity assumption that $\nu \geq \nu_{1} \geq \nu_{0}\left(\lambda, \gamma, \mathbf{u}_{0}, \phi_{0}\right)$.

(b) Assume that (A1), (A2), (F1)-(F3) are satisfied. For any $\mathbf{u}_{0} \in V, \phi_{0} \in \Phi_{2}$, system (1.1)-(1.6) admits a unique global strong solution $(\mathbf{u}, \phi)$ provided that the viscosity and mobility are properly large such that $\nu \geq \nu_{1} \geq \nu_{0}\left(\lambda, \mathbf{u}_{0}, \phi_{0}\right)$ and $\gamma \geq \gamma_{1} \geq$ $\gamma_{0}\left(\lambda, \mathbf{u}_{0}, \phi_{0}\right)$.

Moreover, for both cases (a) and (b), the uniform estimate holds for all $t \geq 0$,

$$
\|\mathbf{u}(t)\|_{V}^{2}+\|\phi(t)\|_{\Phi_{2}}^{2} \leq C,
$$

where $C$ is a constant depending on $\left\|\mathbf{u}_{0}\right\|_{V},\left\|\phi_{0}\right\|_{\Phi_{2}}$ and $|\Omega|$. 
4. Global existence of strong solutions for near equilibrium initial data

In this section, we try to derive a new higher order energy inequality, which will provide us information about the asymptotic behavior of global strong solutions. We also show the existence of global strong solutions for near equilibrium initial data. As before, we shall just perform the calculation for smooth solutions and it can be justified by proper approximation procedure.

Define

$$
\begin{aligned}
& \mathscr{A}(t)=\|\nabla \mathbf{u}(t)\|^{2}+\|\nabla \mu(t)\|^{2}, \\
& \mathscr{B}(t)=\nu_{1}\|S \mathbf{u}(t)\|^{2}+\gamma_{1}\|\nabla \Delta \mu(t)\|^{2} .
\end{aligned}
$$

Lemma 4.1. For the global strong solutions obtained in Proposition 2.5(a) (2-D) or Theorem 3.2 (3-D), there holds

$$
\frac{d}{d t} \mathscr{A}(t)+\mathscr{B}(t) \leq C \mathscr{A}^{2}(t)+C, \quad \forall t>0
$$

where constant $C$ depends on $\lambda, \gamma_{1}, \nu_{1},\left\|\mathbf{u}_{0}\right\|_{V},\left\|\phi_{0}\right\|_{\Phi_{2}}$, and $|\Omega|$.

Proof. Here we only provide a proof for the 3 -D case. For the 2-D case, the proof can be carried out in a similar way with minor modifications. A direct calculation yields that

$$
\begin{aligned}
& \frac{1}{2} \frac{d}{d t}\left(\|\nabla \mathbf{u}\|^{2}+\|\nabla \mu\|^{2}\right)+\int_{\Omega} \nu(\phi)|S \mathbf{u}|^{2} d x+\int_{\Omega} \gamma(\phi)|\nabla \Delta \mu|^{2} d x \\
= & -((\mathbf{u} \cdot \nabla) \mathbf{u}, S \mathbf{u})-(\Delta \phi \nabla \phi, S \mathbf{u})-\left(\nu^{\prime}(\phi) \nabla \phi, \pi S \mathbf{u}\right)+2\left(\nu^{\prime}(\phi) \nabla \phi D(\mathbf{u}), S \mathbf{u}\right) \\
& +(\nabla \Delta \mu, \nabla((\mathbf{u} \cdot \nabla) \phi))-\left(\nabla \Delta \mu, \gamma^{\prime \prime}(\phi)|\nabla \phi|^{2} \nabla \mu+\gamma^{\prime}(\phi) \nabla^{2} \phi \nabla \mu\right) \\
& -\left(\nabla \Delta \mu, \gamma^{\prime}(\phi) \nabla \phi \nabla^{2} \mu+\gamma^{\prime}(\phi) \nabla \phi \Delta \mu\right)-\left(\Delta \mu, f^{\prime}(\phi) \gamma^{\prime}(\phi) \nabla \phi \nabla \mu\right) \\
& +\left(\Delta \mu, f^{\prime}(\phi)(\mathbf{u} \cdot \nabla) \phi\right)-\left(\Delta \mu, f^{\prime}(\phi) \gamma(\phi) \Delta \mu\right) \\
= & I_{1}+I_{2}+\cdots+I_{10} .
\end{aligned}
$$

We now estimate $I_{1}, \ldots, I_{10}$ term by term. The following inequalities in 3 -D case will be frequently used in the subsequent proofs (cf. [3, 38]):

$$
\begin{aligned}
\|\mathbf{u}\|_{L^{\infty}} & \leq C\|\Delta \mathbf{u}\|^{\frac{1}{2}}\|\mathbf{u}\|_{L^{6}}^{\frac{1}{2}}+C\|\mathbf{u}\|_{L^{6}} \leq C\|\Delta \mathbf{u}\|^{\frac{1}{2}}\|\nabla \mathbf{u}\|^{\frac{1}{2}}+C\|\nabla \mathbf{u}\| \\
\|\phi\|_{L^{\infty}} & \leq C\|\phi\|_{H^{1}}^{\frac{1}{2}}\|\phi\|_{H^{2}}^{\frac{1}{2}} \\
\|\nabla \phi\|_{L^{4}} & \leq C\left\|\nabla^{2} \phi\right\|^{\frac{3}{4}}\|\nabla \phi\|^{\frac{1}{4}}+C\|\nabla \phi\| \\
\|\nabla \phi\|_{L^{\infty}} & \leq C\|\nabla \phi\|_{L^{6}}^{\frac{1}{2}}\|\nabla \Delta \phi\|^{\frac{1}{2}}+C\|\nabla \phi\|_{L^{6}} \\
\left\|\nabla^{2} \phi\right\|_{L^{3}} & \leq C\|\nabla \Delta \phi\|^{\frac{1}{2}}\left\|\nabla^{2} \phi\right\|^{\frac{1}{2}}+C\left\|\nabla^{2} \phi\right\| \\
\|\Delta \phi\|_{L^{4}} & \leq C\|\nabla \Delta \phi\|^{\frac{3}{4}}\|\Delta \phi\|^{\frac{1}{4}}+C\|\Delta \phi\| \\
\|\nabla \mu\|_{L^{\infty}} & \leq C\|\nabla \mu\|^{\frac{1}{4}}\|\nabla \Delta \mu\|^{\frac{3}{4}}+C\|\nabla \mu\| \\
\|\Delta \mu\| & \leq C\|\nabla \mu\|^{\frac{1}{2}}\|\nabla \Delta \mu\|^{\frac{1}{2}}+C\|\nabla \mu\|
\end{aligned}
$$

Besides, from the definition of $\mu$ and uniform estimate (2.3), we can see that

$$
\begin{aligned}
\|\nabla \Delta \phi\| & \leq\|\nabla \mu\|+\|\nabla f(\phi)\| \leq\|\nabla \mu\|+C\left(\|\phi\|_{L^{\infty}}^{2}+1\right)\|\nabla \phi\| \\
& \leq\|\nabla \mu\|+C\left(\|\phi\|_{L^{6}}^{\frac{3}{4}}\|\nabla \Delta \phi\|^{\frac{1}{4}}+\|\phi\|_{L^{6}}\right)^{2}\|\nabla \phi\|+C\|\nabla \phi\|
\end{aligned}
$$




$$
\leq\|\nabla \mu\|+C\left(\|\nabla \Delta \phi\|^{\frac{1}{2}}+1\right) \leq \frac{1}{2}\|\nabla \Delta \phi\|+\|\nabla \mu\|+C .
$$

Namely,

$$
\|\nabla \Delta \phi\| \leq 2\|\nabla \mu\|+C,
$$

where $C$ depends on $|\Omega|,\left\|\mathbf{u}_{0}\right\|$ and $\left\|\phi_{0}\right\|_{H^{1}}$.

Always keeping the uniform estimate $(2.6) /(3.25)$ in mind, we can obtain

$$
\begin{aligned}
& \left|I_{1}\right| \leq\|\mathbf{u}\|_{L^{\infty}}\|\nabla \mathbf{u}\|\|S \mathbf{u}\| \leq C\|\nabla \mathbf{u}\|^{\frac{3}{2}}\|S \mathbf{u}\|^{\frac{3}{2}}+C\|\nabla \mathbf{u}\|^{2}\|S \mathbf{u}\| \\
& \leq \varepsilon\|S \mathbf{u}\|^{2}+C(\varepsilon)\left(\|\nabla \mathbf{u}\|^{6}+\|\nabla \mathbf{u}\|^{2}\right), \\
& \left|I_{2}\right| \leq\|S \mathbf{u}\|\|\Delta \phi\|\|\nabla \phi\|_{L^{\infty}} \leq\|S \mathbf{u}\|\|\Delta \phi\|\left(C\|\nabla \phi\|_{L^{6}}^{\frac{1}{2}}\|\nabla \Delta \phi\|^{\frac{1}{2}}+C\|\nabla \phi\|_{L^{6}}\right) \\
& \leq C\|S \mathbf{u}\|\left(\|\nabla \Delta \phi\|^{\frac{1}{2}}+1\right) \leq \varepsilon\|S \mathbf{u}\|^{2}+C(\varepsilon)(\|\nabla \Delta \phi\|+1) \\
& \leq \varepsilon\|S \mathbf{u}\|^{2}+C(\varepsilon)\|\nabla \mu\|^{2}+C, \\
& \left|I_{3}\right| \leq C\|\nabla \phi\|_{L^{\infty}}\|\pi\|\|S \mathbf{u}\| \leq C\|\nabla \phi\|_{L^{\infty}}\|\mathbf{u}\|_{V}\|S \mathbf{u}\| \\
& \leq C\left(C\|\nabla \phi\|_{L^{6}}^{\frac{1}{2}}\|\nabla \Delta \phi\|^{\frac{1}{2}}+C\|\nabla \phi\|_{L^{6}}\right)\|\nabla \mathbf{u}\|\|S \mathbf{u}\| \\
& \leq \varepsilon\|S \mathbf{u}\|^{2}+C(\varepsilon)\|\nabla \mu\|^{2}+C(\varepsilon)\|\nabla \mathbf{u}\|^{4}+C(\varepsilon)\|\nabla \mathbf{u}\|^{2}, \\
& \left|I_{4}\right| \leq C\|\nabla \phi\|_{L^{\infty}}\|D(\mathbf{u})\|\|S \mathbf{u}\| \leq C\left(\|\nabla \Delta \phi\|^{\frac{1}{2}}+1\right)\|\nabla \mathbf{u}\|\|S \mathbf{u}\| \\
& \leq \varepsilon\|S \mathbf{u}\|^{2}+C(\varepsilon)\|\nabla \mu\|^{2}+C(\varepsilon)\|\nabla \mathbf{u}\|^{4}+C(\varepsilon)\|\nabla \mathbf{u}\|^{2}, \\
& \left|I_{5}\right|=|(\nabla \Delta \mu, \nabla \mathbf{u} \nabla \phi+(\mathbf{u} \cdot \nabla) \nabla \phi)| \\
& \leq\|\nabla \Delta \mu\|\left(\|\nabla \mathbf{u}\|\|\nabla \phi\|_{L^{\infty}}+\|\mathbf{u}\|_{L^{6}}\left\|\nabla^{2} \phi\right\|_{L^{3}}\right) \\
& \leq C\|\nabla \Delta \mu\|\|\nabla \mathbf{u}\|\left(\left\|\nabla^{2} \phi\right\|^{\frac{1}{2}}\|\nabla \Delta \phi\|^{\frac{1}{2}}+\|\nabla \phi\|_{L^{6}}+\left\|\nabla^{2} \phi\right\|\right) \\
& \leq C\|\nabla \Delta \mu\|\|\nabla \mathbf{u}\|\left(\|\nabla \Delta \phi\|^{\frac{1}{2}}+1\right) \\
& \leq \varepsilon\|\nabla \Delta \mu\|^{2}+C(\varepsilon)\|\nabla \mu\|^{2}+C(\varepsilon)\|\nabla \mathbf{u}\|^{4}+C(\varepsilon)\|\nabla \mathbf{u}\|^{2}, \\
& \left|I_{6}\right| \leq C\|\nabla \Delta \mu\|\|\nabla \phi\|_{L^{\infty}}^{2}\|\nabla \mu\|+C\|\nabla \Delta \mu\|\left\|\nabla^{2} \phi\right\|\|\| \nabla \mu \|_{L^{\infty}} \\
& \leq C\|\nabla \Delta \mu\|\left(C\|\nabla \phi\|_{L^{6}}^{\frac{1}{2}}\|\nabla \Delta \phi\|^{\frac{1}{2}}+C\|\nabla \phi\|_{L^{6}}\right)^{2}\|\nabla \mu\| \\
& +C\|\nabla \Delta \mu\|\left(C\|\nabla \mu\|^{\frac{1}{4}}\|\nabla \Delta \mu\|^{\frac{3}{4}}+C\|\nabla \mu\|\right) \\
& \leq C\|\nabla \Delta \mu\|(\|\nabla \Delta \phi\|+1)\|\nabla \mu\|+C\|\nabla \Delta \mu\|^{\frac{7}{4}}\|\nabla \mu\|^{\frac{1}{4}}+C\|\nabla \Delta \mu\|\|\nabla \mu\| \\
& \leq \varepsilon\|\nabla \Delta \mu\|^{2}+C(\varepsilon)\|\nabla \mu\|^{4}+C(\varepsilon)\|\nabla \mu\|^{2}, \\
& \left|I_{7}\right| \leq C\|\nabla \Delta \mu\|\|\nabla \phi\|_{L^{\infty}}\|\Delta \mu\| \\
& \leq C\|\nabla \Delta \mu\|\left(\|\nabla \Delta \phi\|^{\frac{1}{2}}+1\right)\left(C\|\nabla \mu\|^{\frac{1}{2}}\|\nabla \Delta \mu\|^{\frac{1}{2}}+C\|\nabla \mu\|\right) \\
& \leq \varepsilon\|\nabla \Delta \mu\|^{2}+C(\varepsilon)\|\nabla \mu\|^{4}+C(\varepsilon)\|\nabla \mu\|^{3}+C(\varepsilon)\|\nabla \mu\|^{2}, \\
& \left|I_{8}\right| \leq C\left\|f^{\prime}(\phi)\right\|_{L^{\infty}}\|\Delta \mu\|\|\nabla \phi\|_{L^{\infty}}\|\nabla \mu\| \\
& \leq C\left(\|\phi\|_{L^{\infty}}\right)\left(C\|\nabla \mu\|^{\frac{1}{2}}\|\nabla \Delta \mu\|^{\frac{1}{2}}+C\|\nabla \mu\|\right)\left(\|\nabla \Delta \phi\|^{\frac{1}{2}}+1\right)\|\nabla \mu\| \\
& \leq \varepsilon\|\nabla \Delta \mu\|^{2}+C(\varepsilon)\|\nabla \mu\|^{3}+C(\varepsilon)\|\nabla \mu\|^{2}+C, \\
& \left|I_{9}\right| \leq\|\Delta \mu\|\left\|f^{\prime}(\phi)\right\|_{L^{\infty}}\|\mathbf{u}\|_{L^{4}}\|\nabla \phi\|_{L^{4}} \\
& \leq C\left(\|\phi\|_{L^{\infty}}\right)\left(C\|\nabla \mu\|^{\frac{1}{2}}\|\nabla \Delta \mu\|^{\frac{1}{2}}+C\|\nabla \mu\|\right)\|\nabla \mathbf{u}\|\left(\|\nabla \phi\|^{\frac{1}{4}}\left\|\nabla^{2} \phi\right\|^{\frac{3}{4}}+\|\nabla \phi\|\right)
\end{aligned}
$$




$$
\begin{aligned}
& \leq \varepsilon\|\nabla \Delta \mu\|^{2}+C(\varepsilon)\|\nabla \mu\|^{2}+C(\varepsilon)\|\nabla \mathbf{u}\|^{2}, \\
\left|I_{10}\right| & \leq\|\gamma(\phi)\|_{L^{\infty}}\left\|f^{\prime}(\phi)\right\|_{L^{\infty}}\|\Delta \mu\|^{2} \leq C\left(\|\phi\|_{L^{\infty}}\right)\|\Delta \mu\|^{2} \\
& \leq C\left(\|\nabla \mu\|^{\frac{1}{2}}\|\nabla \Delta \mu\|^{\frac{1}{2}}+C\|\nabla \mu\|\right)^{2} \\
& \leq \varepsilon\|\nabla \Delta \mu\|^{2}+C(\varepsilon)\|\nabla \mu\|^{2} .
\end{aligned}
$$

Combining all these estimates, and taking $\varepsilon>0$ sufficiently small, we conclude from $(2.6) /(3.25)$ that

$$
\frac{d}{d t}\left(\|\nabla \mathbf{u}\|^{2}+\|\nabla \mu\|^{2}\right)+\nu_{1}\|S \mathbf{u}\|^{2}+\gamma_{1}\|\nabla \Delta \mu\|^{2} \leq C\|\nabla \mathbf{u}\|^{4}+C\|\nabla \mu\|^{4}+C\|\nabla \mu\|^{2}+C,
$$

where the constant $C$ depends on $\gamma_{1}, \lambda, \nu_{1},\left\|\mathbf{u}_{0}\right\|_{V},\left\|\phi_{0}\right\|_{\Phi_{2}}$, and $|\Omega|$.

The proof is complete.

Based on Lemma 4.1, we can deduce the following result:

Lemma 4.2. The global strong solution $(\mathbf{u}, \phi)$ obtained in Proposition 2.5 (2-D) or Theorem 3.2 has the following properties

$$
\begin{gathered}
\lim _{t \rightarrow+\infty}\left(\|\nabla \mathbf{u}(t)\|^{2}+\|\nabla \mu(t)\|^{2}\right)=0, \\
\|\mathbf{u}\|_{V}+\|\phi(t)\|_{H^{3}} \leq C, \quad t \geq 1,
\end{gathered}
$$

where the constant $C$ depends on $\gamma_{1}, \lambda, \nu_{1},\left\|\mathbf{u}_{0}\right\|_{V},\left\|\phi_{0}\right\|_{\Phi_{2}}$, and $|\Omega|$.

Proof. Lemma 4.1 tells us that

$$
\frac{d}{d t} \mathscr{A}(t) \leq C \mathscr{A}^{2}(t)+C \text { for all } t>0
$$

On the other hand, from the basic energy law (2.2), there exists a constant $M$ depending on $\left\|\mathbf{u}_{0}\right\|,\left\|\phi_{0}\right\|_{H^{1}}$, and $|\Omega|$ such that

$$
\int_{0}^{+\infty} \mathscr{A}(t) d t \leq M
$$

Applying [40, Lemma 6.2.1], we conclude that

$$
\begin{gathered}
\mathscr{A}(t) \leq C \text { for all } t \geq 1, \\
\lim _{t \rightarrow+\infty} \mathscr{A}(t)=0 .
\end{gathered}
$$

(2.6)/(3.25), (4.13), and (4.28) imply (4.25). The proof is complete.

Comparing with the stability result in [3], we have the following conclusion, which will be helpful to understand the asymptotic behavior of global solutions in the 3-D case (cf. $[25,19,36,37])$.

Theorem 4.3. Assume $n=3, \mathbf{u}_{0} \in V, \phi_{0} \in \Phi_{2} \cap H^{3}(\Omega)$. For any $R \in(0, \infty)$, whenever

$$
\mathscr{A}(0)=\|\nabla \mathbf{u}(0)\|^{2}+\|\nabla \mu(0)\|^{2} \leq R,
$$


there is a small constant $\varepsilon_{0} \in(0,1)$ depending only on $\nu_{1}, \lambda, \gamma_{1}, \Omega, R$, and $f$ with the following property: either

(i) Problem (1.1)-(1.6) has a unique global strong solution $(\mathbf{u}, \phi)$ in $\Omega \times(0,+\infty)$

or

(ii) there is a $T_{*} \in(0,+\infty)$ such that

$$
\widetilde{E}\left(T_{*}\right)<\widetilde{E}(0)-\varepsilon_{0},
$$

where (cf. (2.1))

$$
\widetilde{E}(t):=E(\mathbf{u}(t), \phi(t))=\frac{1}{2}\|\mathbf{u}(t)\|^{2}+\frac{1}{2}\|\nabla \phi(t)\|^{2}+\int_{\Omega} F(\phi(t)) d x .
$$

Moreover, in case (i), the global strong solution has the same properties as in Lemma 4.2.

Proof. The proof follows from the idea in [19]. Based on the uniform estimate Lemma 2.3, we can proceed as Lemma 4.1 but using different interpolation inequalities and Sobolev embedding theorems to conclude that (we omit the details here)

$$
\frac{d}{d t} \mathscr{A}(t) \leq C_{*}\left(\mathscr{A}(t)^{3}+1\right),
$$

where $C_{*}$ is a constant that only depends on $\nu_{1}, \lambda, \gamma_{1}, f,|\Omega|,\left\|\mathbf{u}_{0}\right\|$ and $\left\|\phi_{0}\right\|_{H^{1}}$.

If the initial data satisfies (4.30), applying the standard argument in ODE theory to (4.31) yields that there exists a $t_{0}=t_{0}\left(R, C_{*}\right)>0$ such that $\mathscr{A}(t)$ is bounded on $\left[0, t_{0}\right]$. The bound only depends on $t_{0}, R$ and $C_{*}$. This fact together with (4.13) and the argument in [3] implies the local existence of a unique strong solution to problem (1.1)-(1.6) in the time interval $\left[0, t_{0}\right]$.

If (ii) is not true, we have

$$
\widetilde{E}(t) \geq \widetilde{E}(0)-\varepsilon_{0} \text { for all } t \geq 0 .
$$

From the basic energy law (2.2), we infer that

$$
\int_{0}^{\infty}\left(2 \nu_{1}\|D(\mathbf{u}(t))\|^{2}+\gamma_{1}\|\nabla \mu(t)\|^{2}\right) d t \leq \varepsilon_{0} .
$$

In particular,

$$
\int_{\frac{t_{0}}{2}}^{t_{0}}\left(2 \nu_{1}\|D(\mathbf{u}(t))\|^{2}+\gamma_{1}\|\nabla \mu(t)\|^{2}\right) d t \leq \varepsilon_{0} .
$$

Hence, there exists a $t_{*} \in\left[\frac{t_{0}}{2}, t_{0}\right]$ such that

$$
2 \nu_{1}\left\|D\left(\mathbf{u}\left(t_{*}\right)\right)\right\|^{2}+\gamma_{1}\left\|\nabla \mu\left(t_{*}\right)\right\|^{2} \leq \frac{2 \varepsilon_{0}}{t_{0}} .
$$

From the Korn's inequality (cf. Lemma 2.2), we infer that

$$
\nu_{1}\left\|\nabla \mathbf{u}\left(t_{*}\right)\right\|^{2}+\gamma_{1}\left\|\nabla \mu\left(t_{*}\right)\right\|^{2} \leq \frac{2 \varepsilon_{0}}{t_{0}} .
$$


Let $\varepsilon_{0}$ satisfy

$$
\frac{2 \varepsilon_{0}}{\min \left\{\gamma_{1}, \nu_{1}\right\} t_{0}} \leq R .
$$

Then we have $\mathscr{A}\left(t_{*}\right) \leq R$. Taking $t_{*}$ as the initial time, we infer from the above argument that $\mathscr{A}(t)$ is bounded at least on $\left[0, \frac{3 t_{0}}{2}\right] \subset\left[0, t_{*}+t_{0}\right]$. Moreover, the bound of $\mathscr{A}(t)$ remains the same as that on $\left[0, t_{0}\right]$. Furthermore, the bounds of $\|u\|_{L^{\infty}\left(t_{*}, t_{*}+t_{0} ; V\right)}$, $\|u\|_{L^{2}\left(t_{*}, t_{*}+t_{0} ; V_{2}\right)},\|\phi\|_{L^{\infty}\left(t_{*}, t_{*}+t_{0} ; \Phi_{2}\right)}$, and $\|\phi\|_{L^{2}\left(t_{*}, t_{*}+t_{0} ; \Phi_{4}\right)}$ are also the same as those in the time interval $\left[0, t_{0}\right]$. Hence we can extend the local unique strong solution step by step to infinity such that

$$
\mathscr{A}(t) \leq C \text { for all } t \geq 0,
$$

where $C$ is uniform in time. (4.24) follows from (4.31), (4.33) and (4.27). Combining Lemma 2.3 and (4.13) with (4.33), we conclude (4.25). The proof is complete.

Denote

$$
\mathcal{E}(\phi)=\frac{1}{2}\|\nabla \phi\|^{2}+\int_{\Omega} F(\phi) d x
$$

Then we have a "stability" result for the near equilibrium initial data.

COROLlary 4.4. Suppose $n=3$. Let $\phi^{*} \in \mathcal{D}$ be an absolute minimizer of $\mathcal{E}(\phi)$ in the sense that $\mathcal{E}\left(\phi^{*}\right) \leq \mathcal{E}(\phi)$ for all $\phi \in \mathcal{D}$. For any $\mathbf{u}_{0} \in V, \phi_{0} \in \Phi_{2} \cap H^{3}(\Omega)$, there is a constant $\sigma$ which may depend on $\lambda, \gamma_{1}, \nu_{1}, f$ and $\left\|\mathbf{u}_{0}\right\|_{V},\left\|\phi_{0}\right\|_{H^{3}}$, such that if $\left\|\mathbf{u}_{0}\right\|+$ $\left\|\phi_{0}-\phi^{*}\right\|_{H^{1}}<\sigma$, then problem (1.1)-(1.6) admits a unique global strong solution which enjoys the same properties as in Lemma 4.2.

Proof. Without loss of generality, we assume that $\sigma \leq 1$. Since $\phi^{*}$ is the absolute minimizer of functional $\mathcal{E}$, from our assumption, we can show that for all $t \geq 0, \widetilde{E}(0)-$ $\widetilde{E}(t) \leq \widetilde{E}(0)-\mathcal{E}\left(\phi^{*}\right) \leq K_{1} \sigma$, where $K_{1}$ is a positive constant that only depends on $\phi_{0}$, $\phi_{*}, \lambda, f$, but not on $\sigma$ (cf. [37]). Besides, set $K_{2}:=\|\nabla \mathbf{u}(0)\|^{2}+\|\nabla \mu(0)\|^{2}<\infty$. We take $R=K_{2}, \varepsilon_{0}=K_{1} \sigma$, and choose

$$
\sigma=\min \left\{1, \frac{K_{2}}{2 K_{1}} \min \left\{\gamma_{1}, \nu_{1}\right\} t_{0}\left(K_{2}, C_{*}\right)\right\} .
$$

Since now $\widetilde{E}(0)-\widetilde{E}(t) \leq \varepsilon_{0}$ for all $t \geq 0$, we know that case (ii) in Theorem 4.3 will not take place. As a consequence, the conclusion follows from Theorem 4.3.

\section{Long-time behavior of global strong solutions}

In this section, for the global strong solutions $(\mathbf{u}, \phi)$ obtained in Proposition 2.5(a) (2-D), Theorem 3.2, and Corollary $4.4(3-\mathrm{D})$, we prove the convergence to equilibrium as well as estimates on the convergence rate.

5.1. Convergence to equilibrium. The $\omega$-limit set of $\left(\mathbf{u}_{0}, \phi_{0}\right) \in V \times \Phi_{2}$ is defined as follows:

$$
\begin{aligned}
\omega\left(\mathbf{u}_{0}, \phi_{0}\right)= & \left\{\left(\mathbf{u}_{\infty}(x), \phi_{\infty}(x)\right) \mid \text { there exists }\left\{t_{n}\right\} \nearrow \infty\right. \text { such that } \\
& \left.\left(\mathbf{u}\left(t_{n}\right), \phi\left(t_{n}\right)\right) \rightarrow\left(\mathbf{u}_{\infty}, \phi_{\infty}\right) \text { in } V \times \Phi_{2}, \text { as } t_{n} \rightarrow+\infty\right\} .
\end{aligned}
$$

Let

$$
\mathcal{S}=\{(0, \phi) \mid-\Delta \phi+f(\phi)=\text { Const. }=m(f(\phi)), \text { a.e. in } \Omega, \quad \phi \in \mathcal{D}\}
$$


We can prove that

Proposition 5.1. Under the assumptions in Theorem 1.1-1.3, the $\omega$-limit set $\omega\left(\mathbf{u}_{0}, \phi_{0}\right)$ is a nonempty compact subset in $V \times \Phi_{2}$. Besides, all asymptotic limiting points $\left(\mathbf{u}_{\infty}, \phi_{\infty}\right)$ of problem (1.1)-(1.6) belong to $\mathcal{S}$, i.e., $\omega\left(\mathbf{u}_{0}, \phi_{0}\right) \subset \mathcal{S}$.

Proof. Since our system admits a Lyapunov functional $E(\mathbf{u}, \phi)$, the proof follows from the argument in [1]. Or we can simply proceed as follows. Equation (4.24) and the Poincaré inequality indicate that

$$
\lim _{t \rightarrow+\infty}\|\mathbf{u}(t)\|_{V}=0
$$

namely, $\mathbf{u}_{\infty}=0$.

Due to (4.25) and the compact embedding $H^{3}(\Omega) \hookrightarrow \Phi_{2}$, there exist a function $\phi_{\infty} \in \Phi_{2}$ and an increasing unbounded sequence $\left\{t_{n}\right\}_{n=1}^{\infty}$ such that

$$
\lim _{t_{n} \rightarrow+\infty}\left\|\phi\left(t_{n}\right)-\phi_{\infty}\right\|_{\Phi_{2}}=0 \text {. }
$$

We infer from Proposition 2.5, Theorem 3.2, Lemma 4.2, and Corollary 4.4 that for all the cases we consider, there holds $\|\nabla \mu(t)\|=\|\nabla(-\Delta \phi+f(\phi))(t)\| \rightarrow 0$ as $t \rightarrow \infty$. Then Poincaré inequality implies that $\|-\Delta \phi(t)+f(\phi(t))-m(f(\phi(t)))\| \rightarrow 0$ as $t \rightarrow \infty$. This and (5.2) yield that $\left\|-\Delta \phi_{\infty}+f\left(\phi_{\infty}\right)-m\left(f\left(\phi_{\infty}\right)\right)\right\|=0$. The proof is complete. $\square$

From the above discussion, we have already known the convergence of velocity field $\mathbf{u}$ (cf. (5.1)). Next, we discuss the convergence of $\phi(t)$. Due to Proposition 5.1, there exists an equilibrium $\phi_{\infty}$ satisfying (1.10) and an increasing sequence $\left\{t_{n}\right\}_{n=1}^{\infty}$ such that

$$
\lim _{t_{n} \rightarrow+\infty}\left\|\phi\left(t_{n}\right)-\phi_{\infty}\right\|_{\Phi_{2}}=0
$$

We shall apply the Eojasiewicz-Simon approach to prove the convergence of $\phi(t)$ to $\phi_{\infty}$ as $t$ goes to infinity. First, we introduce the following Łojasiewicz-Simon type inequality. Let $\mathrm{P}: L^{2}(\Omega) \mapsto L_{0}^{2}(\Omega)$ be a projection operator such that for any $u \in L^{2}(\Omega)$, $\mathrm{P} u=u-m(u)$. We have

LEMma 5.2 (Lojasiewicz-Simon Inequality). Let $\phi_{\infty} \in \mathcal{D}$ be a solution to (1.10). Then there exist constants $\theta \in\left(0, \frac{1}{2}\right)$ and $\beta>0$ depending on $\phi_{\infty}$ such that for any $w \in \mathcal{D}$ satisfying $\left\|w-\phi_{\infty}\right\|_{H^{2}}<\beta$, it holds

$$
\|\mathrm{P}(-\Delta w+f(w))\| \geq\left|\mathcal{E}(w)-\mathcal{E}\left(\phi_{\infty}\right)\right|^{1-\theta} .
$$

Proof. The proof follows from the general result [7] (cf. also [24, 32]. For related results concerning singular free energy density, cf. $[1,2])$.

It follows from $(2.2)$ that $E(\mathbf{u}, \phi)$ is non-negative and decreasing in time. Moreover, $E(\mathbf{u}, \phi) \geq \mathcal{E}\left(\phi_{\infty}\right)$, for all $t>0$. As a result, it has a finite limit as time goes to infinity. It follows from (5.1) and (5.3) that

$$
\lim _{t_{n} \rightarrow+\infty} E\left(\mathbf{u}\left(t_{n}\right), \phi\left(t_{n}\right)\right)=\mathcal{E}\left(\phi_{\infty}\right) .
$$

Equations (1.3) and (4.25) imply that (cf. also [1, 24])

$$
\left\|\phi_{t}\right\|_{H^{-1}} \leq\|(\mathbf{u} \cdot \nabla) \phi\|_{H^{-1}}+\|\gamma(\phi) \nabla \mu\| \leq\|\mathbf{u}\|_{L^{6}}\|\nabla \phi\|_{L^{\frac{3}{2}}}+C\left(\|\phi\|_{L^{\infty}}\right)\|\nabla \mu\|
$$




$$
\leq C(\|\nabla \mathbf{u}\|+\|\nabla \mu\|) .
$$

Hence, if there is a $t_{1} \in \mathbb{R}^{+}$such that $E\left(\mathbf{u}\left(t_{1}\right), \phi\left(t_{1}\right)\right)=\mathcal{E}\left(\phi_{\infty}\right)$, then $\|\nabla \mathbf{u}(t)\|=$ $\|\nabla \mu(t)\|=0$ for all $t \geq t_{1}$ by virtue of (2.2) and Lemma 2.2. Together with (5.6), it implies that $\phi$ is independent of time for all $t \geq t_{1}$. From (5.3), we conclude that

$$
\lim _{t \rightarrow+\infty}\left\|\phi(t)-\phi_{\infty}\right\|_{\Phi_{2}}=0 .
$$

Therefore, we only need to consider the case that $E(\mathbf{u}(t), \phi(t))>\mathcal{E}\left(\phi_{\infty}\right)$ for all $t \geq$ 0 . By a contradiction argument introduced in [17], we can show that there is a $t_{0}>0$ such that for all $t \geq t_{0}, \phi(t)$ satisfies the condition of Lemma 5.2, i.e., $\left\|\phi(t)-\phi_{\infty}\right\|_{\Phi_{2}}<\beta$ (cf. $[40,17,32,36,35,37]$ for details). Then for the constant $\theta \in\left(0, \frac{1}{2}\right)$ in Lemma 5.2, we apply Lemma 5.2 to obtain

$$
\begin{aligned}
& \left(E(\mathbf{u}, \phi)-\mathcal{E}\left(\phi_{\infty}\right)\right)^{1-\theta} \leq\left(\frac{1}{2}\|\mathbf{u}\|^{2}+\left|\mathcal{E}(\phi)-\mathcal{E}\left(\phi_{\infty}\right)\right|\right)^{1-\theta} \\
\leq & \left(\frac{1}{2}\|\mathbf{u}\|^{2}+\|\mathrm{P}(-\Delta \phi+f(\phi))\|^{\frac{1}{1-\theta}}\right)^{1-\theta} \leq C(\|\nabla \mathbf{u}\|+\|\nabla \mu\|) .
\end{aligned}
$$

From (2.2) and Lemma 2.2, there holds

$$
\begin{aligned}
& -\frac{d}{d t}\left(E(\mathbf{u}, \phi)-\mathcal{E}\left(\phi_{\infty}\right)\right)^{\theta}=-\theta\left(E(\mathbf{u}, \phi)-\mathcal{E}\left(\phi_{\infty}\right)\right)^{\theta-1} \frac{d}{d t} E(\mathbf{u}, \phi) \\
\geq & \frac{C \theta\left(2 \nu_{1}\|D(\mathbf{u})\|^{2}+\gamma_{1}\|\nabla \mu\|^{2}\right)}{\|\nabla \mathbf{u}\|+\|\nabla \mu\|} \geq \frac{C \theta\left(\nu_{1}\|\nabla \mathbf{u}\|^{2}+\gamma_{1}\|\nabla \mu\|^{2}\right)}{\|\nabla \mathbf{u}\|+\|\nabla \mu\|} \\
\geq & \tilde{C}(\|\nabla \mathbf{u}\|+\|\nabla \mu\|), \quad \forall t \geq t_{0},
\end{aligned}
$$

where $\tilde{C}$ is a constant depending on $\left\|\mathbf{u}_{0}\right\|,\left\|\phi_{0}\right\|_{H^{1}}, \Omega, \nu_{1}, \gamma_{1}, \lambda$ and $\theta$. Integrating from $t_{0}$ to $\infty$, we obtain

$$
\int_{t_{0}}^{\infty}(\|\nabla \mathbf{u}(\tau)\|+\|\nabla \mu(\tau)\|) d \tau<+\infty
$$

which together with (5.6) yields

$$
\int_{t_{0}}^{\infty}\left\|\phi_{t}(\tau)\right\|_{H^{-1}} d \tau<+\infty
$$

Thus, we can conclude that $\phi(t)$ converges in $H^{-1}$ as $t \rightarrow+\infty$. This fact together with the compactness in $\Phi_{2}$ and (5.3) indicates that (5.7) holds.

Similar to [1], the uniform estimate (4.25) implies the weak convergence

$$
\phi(t) \rightarrow \phi_{\infty} \quad \text { in } H^{3} \text {, as } t \rightarrow+\infty .
$$

However, the decay of $\mathscr{A}(t)$ (see (4.24)) can tell us more information (cf. also [35, 36, 37]). Since

$$
\left\|\nabla \Delta\left(\phi-\phi_{\infty}\right)\right\| \leq\|\nabla \mu\|+\left\|\nabla\left(f(\phi)-f\left(\phi_{\infty}\right)\right)\right\| \leq\|\nabla \mu\|+C\left\|\phi-\phi_{\infty}\right\|_{H^{1}},
$$

it follows from (4.24) and (5.7) that

$$
\lim _{t \rightarrow+\infty}\left\|\phi(t)-\phi_{\infty}\right\|_{H^{3}}=0 .
$$


5.2. Estimate on the convergence rate. The required estimate on the convergence rate (1.11) is achieved in several steps.

Step 1. $H^{-1}$-estimate for $\phi-\phi_{\infty}$. In view of (5.8) and (5.9), we have

$$
\frac{d}{d t}\left(E(\mathbf{u}, \phi)-\mathcal{E}\left(\phi_{\infty}\right)\right)+C\left(E(\mathbf{u}, \phi)-\mathcal{E}\left(\phi_{\infty}\right)\right)^{2(1-\theta)} \leq 0, t \geq t_{0}
$$

which implies

$$
E(\mathbf{u}, \phi)-\mathcal{E}\left(\phi_{\infty}\right) \leq C(1+t)^{-1 /(1-2 \theta)}, t \geq t_{0} .
$$

Integrating (5.9) from $t$ to $\infty$, (where $t \geq t_{0}$ ), and using (5.6), we obtain

$$
\int_{t}^{\infty}\left\|\phi_{t}\right\|_{H^{-1}} d t \leq C(1+t)^{-\theta /(1-2 \theta)}, t \geq t_{0}
$$

By adjusting the constant $C$ properly (ref. Lemma 2.3), we have

$$
\left\|\phi-\phi_{\infty}\right\|_{H^{-1}} \leq C(1+t)^{-\theta /(1-2 \theta)}, t \geq 0
$$

In order to obtain the same estimates on convergence rate in both higher and lower order norms, the basic idea is to derive suitable energy estimates and proper differential inequalities (cf. [33, 34, 35, 36, 37]).

Step 2. Estimates on $\left\|\phi-\phi_{\infty}\right\|_{H^{1}}$ and $\|\mathbf{u}\|$. The difference function $\phi-\phi_{\infty}$ satisfies

$$
\left\{\begin{array}{l}
\frac{d}{d t}\left(\phi-\phi_{\infty}\right)+(\mathbf{u} \cdot \nabla) \phi=\operatorname{div}(\gamma(\phi) \nabla \tilde{\mu}) \\
\tilde{\mu}=\mu-\mu_{\infty}=-\Delta\left(\phi-\phi_{\infty}\right)+f(\phi)-f\left(\phi_{\infty}\right) \\
\left.\partial_{\mathbf{n}}\left(\phi-\phi_{\infty}\right)\right|_{\Gamma}=\left.\partial_{\mathbf{n}}\left(\mu-\mu_{\infty}\right)\right|_{\Gamma}=0 \\
\int_{\Omega}\left(\phi(x, t)-\phi_{\infty}(x)\right) d x=0
\end{array}\right.
$$

REMARK 5.1. Since $\mu_{\infty}$ is a constant, it is obvious that $\nabla \tilde{\mu}=\nabla \mu$.

Taking the inner product of (1.1) with $\mathbf{u}$ in $L^{2}(\Omega)$, we obtain

$$
\frac{1}{2} \frac{d}{d t}\|\mathbf{u}\|^{2}+2 \int_{\Omega} \nu(\phi)|D(\mathbf{u})|^{2} d x=-(\mathbf{u}, \nabla \phi \Delta \phi) .
$$

Taking the inner product of $\left(\phi-\phi_{\infty}\right)_{t}$ with $\tilde{\mu}$ in $L^{2}(\Omega)$, we have

$$
\begin{aligned}
& \frac{d}{d t}\left[\frac{1}{2}\left\|\nabla\left(\phi-\phi_{\infty}\right)\right\|^{2}+\int_{\Omega} F(\phi) d x-\int_{\Omega} f\left(\phi_{\infty}\right) \phi d x\right]+\int_{\Omega} \gamma(\phi)|\nabla \mu|^{2} d x \\
= & ((\mathbf{u} \cdot \nabla) \phi, \Delta \phi)-((\mathbf{u} \cdot \nabla) \phi, f(\phi))-\left((\mathbf{u} \cdot \nabla) \phi, \Delta \phi_{\infty}-f\left(\phi_{\infty}\right)\right) .
\end{aligned}
$$

Taking the inner product of $\left(-\Delta_{N}\right)^{-1}\left(\phi-\phi_{\infty}\right)_{t}$ with $\phi-\phi_{\infty}$ in $L^{2}(\Omega)$, using $(5.16)$, we obtain

$$
\begin{aligned}
& \frac{1}{2} \frac{d}{d t}\left\|\phi-\phi_{\infty}\right\|_{H^{-1}}^{2}+\int_{\Omega} \gamma(\phi)\left|\nabla\left(\phi-\phi_{\infty}\right)\right|^{2} d x \\
= & -\left(f(\phi)-f\left(\phi_{\infty}\right), \gamma(\phi)\left(\phi-\phi_{\infty}\right)\right)-\left(\nabla\left(\phi-\phi_{\infty}\right), \gamma^{\prime}(\phi) \nabla \phi\left(\phi-\phi_{\infty}\right)\right) \\
& +\left(\tilde{\mu}, \gamma^{\prime}(\phi) \nabla \phi \nabla\left(-\Delta_{N}\right)^{-1}\left(\phi-\phi_{\infty}\right)\right) \\
& -\left(\nabla\left(-\Delta_{N}\right)^{-1}(\mathbf{u} \cdot \nabla) \phi, \nabla\left(-\Delta_{N}\right)^{-1}\left(\phi-\phi_{\infty}\right)\right) .
\end{aligned}
$$


Adding (5.17), (5.18), and (5.19) together, we obtain

$$
\begin{aligned}
& \frac{d}{d t}\left[\frac{1}{2}\|\mathbf{u}\|^{2}+\frac{1}{2}\left\|\phi-\phi_{\infty}\right\|_{H^{-1}}^{2}+\frac{1}{2}\left\|\nabla\left(\phi-\phi_{\infty}\right)\right\|^{2}+\left(\int_{\Omega} F(\phi) d x-\int_{\Omega} F\left(\phi_{\infty}\right) d x\right.\right. \\
& \left.\left.+\int_{\Omega} f\left(\phi_{\infty}\right) \phi_{\infty} d x-\int_{\Omega} f\left(\phi_{\infty}\right) \phi d x\right)\right]+2 \int_{\Omega} \nu(\phi)|D(\mathbf{u})|^{2} d x+\int_{\Omega} \gamma(\phi)|\nabla \mu|^{2} d x \\
& +\int_{\Omega} \gamma(\phi)\left|\nabla\left(\phi-\phi_{\infty}\right)\right|^{2} d x \\
= & -\left(f(\phi)-f\left(\phi_{\infty}\right), \gamma(\phi)\left(\phi-\phi_{\infty}\right)\right)-\left(\nabla\left(\phi-\phi_{\infty}\right), \gamma^{\prime}(\phi) \nabla \phi\left(\phi-\phi_{\infty}\right)\right) \\
& +\left(\tilde{\mu}, \gamma^{\prime}(\phi) \nabla \phi \nabla\left(-\Delta_{N}\right)^{-1}\left(\phi-\phi_{\infty}\right)\right) \\
& -\left(\nabla\left(-\Delta_{N}\right)^{-1}(\mathbf{u} \cdot \nabla) \phi, \nabla\left(-\Delta_{N}\right)^{-1}\left(\phi-\phi_{\infty}\right)\right),
\end{aligned}
$$

where we use the fact that

$$
\begin{gathered}
((\mathbf{u} \cdot \nabla) \phi, f(\phi))=(\mathbf{u}, \nabla F(\phi))=-(\nabla \cdot \mathbf{u}, F(\phi))=0, \\
\left((\mathbf{u} \cdot \nabla) \phi, \Delta \phi_{\infty}-f\left(\phi_{\infty}\right)\right)=-\mu_{\infty}(\mathbf{u}, \nabla \phi)=\mu_{\infty}(\nabla \cdot \mathbf{u}, \phi)=0 .
\end{gathered}
$$

Let

$$
\begin{aligned}
y_{1}(t)= & \frac{1}{2}\|\mathbf{u}\|^{2}+\frac{1}{2}\left\|\phi-\phi_{\infty}\right\|_{H^{-1}}^{2}+\frac{1}{2}\left\|\nabla\left(\phi-\phi_{\infty}\right)\right\|^{2} \\
& +\int_{\Omega}\left(F(\phi)-F\left(\phi_{\infty}\right)+f\left(\phi_{\infty}\right) \phi_{\infty}-f\left(\phi_{\infty}\right) \phi\right) d x .
\end{aligned}
$$

Applying Newton-Leibniz formula $F(u)=F(\psi)+f(\psi)(u-\psi)+\int_{0}^{1} \int_{0}^{1} f^{\prime}(s z u+(1-$ $s z) \psi) z(u-\psi)^{2} d s d z$, we have

$$
\begin{aligned}
& \left|\int_{\Omega}\left(F(\phi)-F\left(\phi_{\infty}\right)+f\left(\phi_{\infty}\right) \phi_{\infty}-f\left(\phi_{\infty}\right) \phi\right) d x\right| \\
\leq & C\left\|\phi-\phi_{\infty}\right\|_{L^{4}}^{2} \leq \frac{1}{4}\left\|\nabla\left(\phi-\phi_{\infty}\right)\right\|^{2}+C\left\|\phi-\phi_{\infty}\right\|^{2} .
\end{aligned}
$$

In a similar way,

$$
\begin{aligned}
& \left|\left(f(\phi)-f\left(\phi_{\infty}\right), \gamma(\phi)\left(\phi-\phi_{\infty}\right)\right)\right| \\
\leq & \|\gamma(\phi)\|_{L^{\infty}}\left|\int_{\Omega} \int_{0}^{1} f^{\prime}\left(s \phi+(1-s) \phi_{\infty}\right)\left(\phi-\phi_{\infty}\right)^{2} d s d x\right| \\
\leq & \varepsilon\left\|\nabla\left(\phi-\phi_{\infty}\right)\right\|^{2}+C(\varepsilon)\left\|\phi-\phi_{\infty}\right\|^{2} .
\end{aligned}
$$

Besides,

$$
\begin{aligned}
\left|\left(\nabla\left(\phi-\phi_{\infty}\right), \gamma^{\prime}(\phi) \nabla \phi\left(\phi-\phi_{\infty}\right)\right)\right| & \leq C\|\nabla \phi\|_{L^{6}}\left\|\nabla\left(\phi-\phi_{\infty}\right)\right\|\left\|\phi-\phi_{\infty}\right\|_{L^{3}} \\
& \leq \varepsilon\left\|\nabla\left(\phi-\phi_{\infty}\right)\right\|^{2}+C(\varepsilon)\left\|\phi-\phi_{\infty}\right\|^{2}
\end{aligned}
$$

From (4.25) and (4.8), using the Poincaré inequality we obtain

$$
\begin{aligned}
\|\tilde{\mu}\| & =\left\|\mu-\mu_{\infty}\right\| \leq C\left(\|\nabla \mu\|+\left|\int_{\Omega}\left(\mu-\mu_{\infty}\right) d x\right|\right) \\
& \leq C\|\nabla \mu\|+\left|\int_{\Omega}\left(f(\phi)-f\left(\phi_{\infty}\right)\right) d x\right|
\end{aligned}
$$




$$
\leq C\|\nabla \mu\|+C\left\|\phi-\phi_{\infty}\right\| \text {. }
$$

As a result,

$$
\begin{aligned}
&\left(\tilde{\mu}, \gamma^{\prime}(\phi) \nabla \phi \nabla\left(-\Delta_{N}\right)^{-1}\left(\phi-\phi_{\infty}\right)\right) \\
& \leq C\|\nabla \phi\|_{L^{\infty}}\|\tilde{\mu}\|\left\|\phi-\phi_{\infty}\right\|_{H^{-1}} \leq C\left(C\|\nabla \mu\|+C\left\|\phi-\phi_{\infty}\right\|\right)\left\|\phi-\phi_{\infty}\right\|_{H^{-1}} \\
& \leq \varepsilon\|\nabla \mu\|^{2}+C(\varepsilon)\left\|\phi-\phi_{\infty}\right\|_{H^{-1}}^{2}+C\left\|\phi-\phi_{\infty}\right\|^{2}, \quad t \geq 1 . \\
& \\
& \quad\left|\left(\nabla\left(-\Delta_{N}\right)^{-1}(\mathbf{u} \cdot \nabla) \phi, \nabla\left(-\Delta_{N}\right)^{-1}\left(\phi-\phi_{\infty}\right)\right)\right| \\
& \leq\|(\mathbf{u} \cdot \nabla) \phi\|_{H^{-1}}\left\|\phi-\phi_{\infty}\right\|_{H^{-1}} \leq\|\nabla \phi\|_{L^{\frac{3}{2}}}\|\mathbf{u}\|_{L^{6}}\left\|\phi-\phi_{\infty}\right\|_{H^{-1}} \\
& \leq C\|\nabla \mathbf{u}\|\left\|\phi-\phi_{\infty}\right\|_{H^{-1}} \leq \varepsilon\|\nabla \mathbf{u}\|^{2}+C(\varepsilon)\left\|\phi-\phi_{\infty}\right\|_{H^{-1}}^{2} .
\end{aligned}
$$

Taking $\varepsilon$ small enough, we conclude from Lemma 2.2 and (5.20)-(5.27) that

$$
\begin{aligned}
& \frac{d}{d t} y_{1}(t)+\frac{\nu_{1}}{2}\|\nabla \mathbf{u}\|^{2}+\frac{\gamma_{1}}{2}\|\nabla \mu\|^{2}+\frac{\gamma_{1}}{2}\left\|\nabla\left(\phi-\phi_{\infty}\right)\right\|^{2} \\
\leq & C\left\|\phi-\phi_{\infty}\right\|^{2}+C\left\|\phi-\phi_{\infty}\right\|_{H^{-1}}^{2}, \quad t \geq 1 .
\end{aligned}
$$

On the other hand, it follows from (5.21) and (5.22) that

$$
y_{1}(t) \leq C\|\nabla \mathbf{u}\|^{2}+\frac{1}{2}\left\|\phi-\phi_{\infty}\right\|_{H^{-1}}^{2}+\frac{3}{4}\left\|\nabla\left(\phi-\phi_{\infty}\right)\right\|^{2}+C\left\|\phi-\phi_{\infty}\right\|^{2}, \quad t \geq 0 .
$$

Moreover, we have

$$
\begin{aligned}
\left\|\phi-\phi_{\infty}\right\|^{2} & \leq C\left\|\phi-\phi_{\infty}\right\|_{H^{-1}}\left\|\phi-\phi_{\infty}\right\|_{H^{1}} \leq C\left\|\phi-\phi_{\infty}\right\|_{H^{-1}}\left\|\nabla\left(\phi-\phi_{\infty}\right)\right\| \\
& \leq \delta\left\|\nabla\left(\phi-\phi_{\infty}\right)\right\|^{2}+C(\delta)\left\|\phi-\phi_{\infty}\right\|_{H^{-1}}^{2}
\end{aligned}
$$

Taking $\delta$ sufficiently small in (5.30), we conclude from (5.28), (5.29), (5.30), and (5.15) that there exists $\alpha_{1}>0$ such that

$$
\frac{d}{d t} y_{1}(t)+\alpha_{1} y_{1}(t) \leq C\left\|\phi-\phi_{\infty}\right\|_{H^{-1}}^{2} \leq C(1+t)^{-2 \theta /(1-2 \theta)}, \quad t \geq 1 .
$$

Therefore, we obtain (cf. [33, 34])

$$
y_{1}(t) \leq C(1+t)^{-2 \theta /(1-2 \theta)}, t \geq 1 .
$$

Noticing (5.29) and adjusting the constant $C$ properly, we have

$$
y_{1}(t) \leq C(1+t)^{-2 \theta /(1-2 \theta)}, t \geq 0 .
$$

From (5.21) and (5.22), we also obtain

$$
y_{1}(t) \geq \frac{1}{2}\|\mathbf{u}\|^{2}+\frac{1}{2}\left\|\phi-\phi_{\infty}\right\|_{H^{-1}}^{2}+\frac{1}{4}\left\|\nabla\left(\phi-\phi_{\infty}\right)\right\|^{2}-C\left\|\phi-\phi_{\infty}\right\|^{2} .
$$

Taking $\delta$ small in (5.30), it follows from (5.33) that there exists a constant $C>0$ such that

$$
y_{1}(t) \geq \frac{1}{2}\|\mathbf{u}\|^{2}+\frac{1}{8}\left\|\nabla\left(\phi-\phi_{\infty}\right)\right\|^{2}-C\left\|\phi-\phi_{\infty}\right\|_{H^{-1}}^{2}
$$


Combining (5.32) with (5.15) and (5.34), we have

$$
\|\mathbf{u}\|+\left\|\phi-\phi_{\infty}\right\|_{H^{1}} \leq C(1+t)^{-\theta /(1-2 \theta)}, t \geq 0
$$

Step 3. Higher-order estimates. In order to obtain a further estimate on convergence rate, we shall revisit Lemma 4.1. For this propose, we only need to redo the estimate of $I_{2}$ (cf. (4.15)) as follows

$$
\begin{aligned}
\left|I_{2}\right| & =\left|\left(\left(\mu-\mu_{\infty}\right) \nabla \phi, S \mathbf{u}\right)-(f(\phi) \nabla \phi, S \mathbf{u})+\left(\mu_{\infty} \nabla \phi, S \mathbf{u}\right)\right| \\
& \leq\|S \mathbf{u}\|\left\|\mu-\mu_{\infty}\right\|\|\nabla \phi\|_{L^{\infty}} \\
& \leq C\|S \mathbf{u}\|\left(\|\nabla \mu\|+\left\|\phi-\phi_{\infty}\right\|\right)\left(C\|\nabla \phi\|_{L^{6}}^{\frac{1}{2}}\|\nabla \Delta \phi\|^{\frac{1}{2}}+C\|\nabla \phi\|_{L^{6}}\right) \\
& \leq C\|S \mathbf{u}\|\left(\|\nabla \Delta \phi\|^{\frac{1}{2}}+1\right)\left(\|\nabla \mu\|+\left\|\phi-\phi_{\infty}\right\|\right) \\
& \leq \varepsilon\|S \mathbf{u}\|^{2}+C(\varepsilon)(\|\nabla \mu\|+1)\left(\|\nabla \mu\|^{2}+\left\|\phi-\phi_{\infty}\right\|^{2}\right) .
\end{aligned}
$$

In the above, we have used the facts $(f(\phi) \nabla \phi, S \mathbf{u})=\left(\mu_{\infty} \nabla \phi, S \mathbf{u}\right)=0$ and estimates (4.13) and (5.25). Collecting the other estimates in the proof of Lemma 4.1 and applying the uniform bounds (4.28), we can improve the inequality (4.3) to obtain

$$
\frac{d}{d t} \mathscr{A}(t) \leq C_{1} \mathscr{A}(t)+C\left\|\phi-\phi_{\infty}\right\|^{2}, \quad \forall t \geq 1
$$

Choose $\kappa>0$ such that

$$
\kappa C_{1} \leq \min \left\{\frac{\nu_{1}}{4}, \frac{\gamma_{1}}{4}\right\}
$$

Multiplying (5.37) by $\kappa$, then adding the resultant to (5.28), we infer from (5.29) and (5.35) that there exists a small constant $\alpha_{2}>0$ such that

$$
\begin{aligned}
& \frac{d}{d t}\left[y_{1}(t)+\kappa \mathscr{A}(t)\right]+\alpha_{2}\left[y_{1}(t)+\kappa \mathscr{A}(t)\right] \leq C\left\|\phi-\phi_{\infty}\right\|^{2} \\
\leq & C(1+t)^{-2 \theta /(1-2 \theta)}, \forall t \geq 1 .
\end{aligned}
$$

Similar to (5.32), we can obtain

$$
y_{1}(t)+\kappa \mathscr{A}(t) \leq C(1+t)^{-2 \theta /(1-2 \theta)}, \forall t \geq 1 .
$$

It follows from (5.32) and (5.39) that

$$
\mathscr{A}(t) \leq C(1+t)^{-2 \theta /(1-2 \theta)}, \forall t \geq 1 .
$$

Namely,

$$
\|\nabla \mathbf{u}(t)\|^{2}+\|\nabla \mu(t)\|^{2} \leq C(1+t)^{-2 \theta /(1-2 \theta)}, \forall t \geq 1 .
$$

By (2.6), the Sobolev embedding theorem and (5.35), we can obtain that

$$
\left\|f(\phi)-f\left(\phi_{\infty}\right)\right\|_{H^{1}} \leq C\left\|\phi-\phi_{\infty}\right\|_{H^{1}} \leq C(1+t)^{-\theta /(1-2 \theta)}, \forall t \geq 1 .
$$

From the definition of the constant $\mu_{\infty}$ (cf. (1.10)), we know that

$$
\begin{aligned}
& -\Delta\left(\phi-\phi_{\infty}\right)=\mu-\mu_{\infty}-f(\phi)+f\left(\phi_{\infty}\right), \\
& \left.\partial_{\mathbf{n}}\left(\phi-\phi_{\infty}\right)\right|_{\Gamma}=0 .
\end{aligned}
$$


Besides, it follows from (5.25), (5.41), and (5.35) that

$$
\left\|\mu-\mu_{\infty}\right\|_{H^{1}} \leq C(1+t)^{-\theta /(1-2 \theta)}, \forall t \geq 1 .
$$

Thus, from the elliptic regularity theorem for problem (5.43)-(5.44), using (5.42), (5.45), and (5.35), we can conclude that

$$
\begin{aligned}
\left\|\phi-\phi_{\infty}\right\|_{H^{3}} & \leq C\left(\left\|\mu-\mu_{\infty}\right\|_{H^{1}}+\left\|f(\phi)-f\left(\phi_{\infty}\right)\right\|_{H^{1}}+\left\|\phi-\phi_{\infty}\right\|\right) \\
& \leq C(1+t)^{-\theta /(1-2 \theta)}, \forall t \geq 1 .
\end{aligned}
$$

We complete the proof.

Acknowledgement. The authors want to thank Prof. C. Liu for his helpful comments and discussions. The research of L. Zhao and H. Huang is partially supported by NSF of China under the Grant No. 10531040. The research of $\mathrm{H}$. Wu is partially supported by China Postdoctoral Science Foundation. The authors are very grateful to the referees for their careful reading and helpful suggestions.

\section{REFERENCES}

[1] H. Abels, On a diffuse interface model for two-phase flows of viscous, incompressible fluids with matched densities, Arch. Rat. Mech. Anal., DOI: 10.1007/s00205-008-0160-2, 2009.

[2] H. Abels and M. Wilke, Convergence to equilibrium for the Cahn-Hilliard equation with a logarithmic free energy, Nonlinear Anal., 67, 3176-3193, 2007.

[3] F. Boyer, Mathematical study of multi-phase flow under shear through order parameter formulation, Asymptot. Anal., 20(2), 175-212, 1999.

[4] P.W. Bates and P.C. Fife, The dynamics of nucleation for the Cahn-Hilliard equation, SIAM J. Appl. Math., 53, 990-1008, 1993.

[5] R. Chill, On the Eojasiewicz-Simon gradient inequality, J. Func. Anal., 201, 572-601, 2003.

[6] X. Chen and M. Kowalczyk, Existence of equilibria for the Cahn-Hilliard equation via local minimizers of the perimeter, Commun. Part. Differ. Equs., 21, 1207-1233, 1996.

[7] H. Gajewski and J. Griepentrog, A descent method for the free energy of multicomponent systems, Discrete Contin. Dyn. Syst. A, 15(2), 505-528, 2006.

[8] C.G. Gal and M. Grasselli, Longtime behavior for a model of homogeneous incompressible two-phase flows, preprint, 2008.

[9] C.G. Gal and M. Grasselli, Asymptotic behavior of a Cahn-Hilliard-Navier-Stokes system in 2D, preprint, 2009.

[10] C.G. Gal and M. Grasselli, Trajectory attractors for binary fluid mixtures in 3D, preprint, 2009.

[11] C.G. Gal and H. Wu, Asymptotic behavior of a Cahn-Hilliard equation with Wentzell boundary conditions and mass conservation, Discrete Contin. Dyn. Syst., 22(4), 1041-1063, 2008.

[12] M. Grinfeld and A. Novick-Cohen, Counting stationary solutions of the Cahn-Hilliard equation by transversality arguments, Proc. Roy. Soc. Edinburgh Sect. A, 125(2), 351-370, 1998.

[13] A. Haraux, Systèmes Dynamiques Dissipatifs et Applications, Masson, Paris, 1991.

[14] A. Haraux and M.A. Jendoubi, Decay estimates to equilibrium for some evolution equations with an analytic nonlinearity, Asymptot. Anal., 26, 21-36, 2001.

[15] C.O. Horgan, Korn's inequalities and their applications in continuum mechanics, SIAM Review, 37(4), 491-511, 1995.

[16] D. Jacqmin, Calculation of two-phase Navier-Stokes flows using phase-field modeling, J. Comput. Phys., 155, 96-127, 1999.

[17] M.A. Jendoubi, A simple unified approach to some convergence theorem of L. Simon, J. Func. Anal., 153, 187-202, 1998.

[18] F.H. Lin and Q. Du, Ginzburg-Landau vortices: dynamics, pinning, and hysteresis, SIAM J. Math. Anal., 28, 1265-1293, 1997.

[19] F.H. Lin and C. Liu, Nonparabolic dissipative system modeling the flow of liquid crystals, Commun. Pure Appl. Math., XLVIII, 501-537, 1995.

[20] C. Liu and J. Shen, A phase field model for the mixture of two incompressible fluids and its approxination by a Fourier-spectral method, Physica D, 179, 211-228, 2003. 
[21] C. Liu and N.J. Walkington, An Eulerian description of fluids containing visco-hyperelastic particles, Arch. Rat. Mech. Anal., 159, 229-252, 2001.

[22] A. Miranville and S. Zelik, Robust exponential attractors for Cahn-Hilliard type equations with singular potentials, Math. Methods Appl. Sci., 27(5), 545-582, 2004.

[23] A. Novick-Cohen and A. Peletier, Steady states of the one-dimensional Cahn-Hilliard equation, Proc. Roy. Soc. Edinburgh Sect. A, 123, 1071-1098, 1993.

[24] P. Rybka and K.H. Hoffmann, Convergence of solutions to Cahn-Hilliard equation, Commun. Part. Differ. Equs., 24(5-6), 1055-1077, 1999.

[25] L. Simon, Asymptotics for a class of nonlinear evolution equation with applicationa to geometric problems, Ann. of Math., 118, 525-571, 1983.

[26] V.N. Starovoitov, On the motion of a two-component fluid in the presence of capillary forces, Mat. Zametki, 62(2), 293-305, 1997.

[27] R. Temam, Navier-Stokes Equations, North-Holland Publishing Comput., (Studies in Mathematics and its Applications; 2), 1977.

[28] R. Temam, Infinite Dimensional Dynamical Systems in Mechanics and Physics, New York, Springer, 1997

[29] J. Wei and M. Winter, Stationary solutions for the Cahn-Hilliard equation, Ann. Inst. H. Poincaré, 15, 459-492, 1998.

[30] J. Wei and M. Winter, On the stationary Cahn-Hilliard equation: interior spike solutions, J. Differ. Equs., 148, 231-267, 1998.

[31] J. Wei and M. Winter, On the stationary Cahn-Hilliard equation: bubble solutions, SIAM J. Math. Anal., 29(6), 1492-1518, 1998.

[32] $\mathrm{H}$. Wu and S. Zheng, Convergence to equibrium for the Cahn-Hilliard equation with dynamic boundary conditions, J. Differ. Equs., 204, 511-531, 2004.

[33] H. Wu, M. Grasselli and S. Zheng, Convergence to equilibrium for a parabolic-hyperbolic phasefield system with Neumann boundary conditions, Math. Models Meth. Appl. Sci., 17(1), $1-29,2007$.

[34] $\mathrm{H} . \mathrm{Wu}$, Convergence to equilibrium for a Cahn-Hilliard model with the Wentzell boundary condition, Asymptot. Anal., 54(1-2), 71-92, 2007.

[35] H. Wu, Long-time behavior for a nonlinear plate equation with thermal memory, J. Math. Anal. Appl., 348, 650-670, 2008.

[36] $\mathrm{H}$. Wu, Long-time behavior for nonlinear hydrodynamic system modeling the nematic liquid crystal flows, Discrete Contin. Dyn. Syst. A, 26(1). 379-396, 2010.

[37] H. Wu, X. Xu and C. Liu, Long-time behavior of hydrodynamic system modeling the nematic liquid crystals, IMA, 2260, preprint, 2009.

[38] L. Zhao, H. Huang and H. Zhang, Strong solutions to a phase-field model for complex fluids, Int. J. Pure Appl. Math., 54(1), 57-73, 2009.

[39] S. Zheng, Asymptotic behavior of solutions to the Cahn-Hilliard equation, Appl. Anal., 23, 165-184, 1986.

[40] S. Zheng, Nonlinear Evolution Equations, Pitman series Monographs and Survey in Pure and Applied Mathematics, 133, Chapman \& Hall/CRC, Boca Raton, Florida, 2004. 